\title{
Post-Newtonian Models of Binary Neutron Stars
}

\author{
James C. Lombardi, Jr. \\ Center for Radiophysics and Space Research, Cornell University, Ithaca, NY 14853 \\ Department of Astronomy, Cornell University, Ithaca, NY 14853
}

Frederic A. Rasio

Department of Physics, MIT 6-201, Cambridge, MA 02139

\author{
Stuart L. Shapiro \\ Departments of Physics and Astronomy, University of Illinois at Urbana-Champaign, 1110 West \\ Green Street, Urbana, IL 61801 \\ National Center For Supercomputing Applications, University of Illinois at Urbana-Champaign, \\ Urbana, IL 61801
}

\begin{abstract}
Using an energy variational method, we calculate quasi-equilibrium configurations of binary neutron stars modeled as compressible triaxial ellipsoids obeying a polytropic equation of state. Our energy functional includes terms both for the internal hydrodynamics of the stars and for the external orbital motion. We add the leading post-Newtonian (PN) corrections to the internal and gravitational energies of the stars, and adopt hybrid orbital terms which are fully relativistic in the test-mass limit and always accurate to PN order. The total energy functional is varied to find quasi-equilibrium sequences for both corotating and irrotational binaries in circular orbits. We examine how the orbital frequency at the innermost stable circular orbit depends on the polytropic index $n$ and the compactness parameter $G M / R c^{2}$. We find that, for a given $G M / R c^{2}$, the innermost stable circular orbit along an irrotational
\end{abstract}


sequence is about $17 \%$ larger than the innermost secularly stable circular orbit along the corotating sequence when $n=0.5$, and $20 \%$ larger when $n=1$. We also examine the dependence of the maximum neutron star mass on the orbital frequency and find that, if PN tidal effects can be neglected, the maximum equilibrium mass increases as the orbital separation decreases.

04.25.Nx,04.30.Db,04.40.Dg,97.60.Jd

Typeset using REVTEX 


\section{INTRODUCTION}

The mergers of neutron star, black hole, and neutron star-black hole binaries provide the most promising sources of gravitational waves for detection by laser interferometers now being constructed, such as LIGO and VIRGO. A variety of additional factors also motivate the study of binary neutron star systems: for example, such systems are known to exist with several having been detected in our Galaxy in the form of binary radio pulsars (eg. [1] and references therein), and coalescing neutron stars may be a source of gamma-ray bursts at cosmological distances [2]. These facts have motivated numerous theoretical studies of the inspiral and coalescence of compact binaries. Until recently, such calculations have involved either post-Newtonian (PN) point mass approximations or purely Newtonian hydrodynamic treatments.

Analytic analyses of the gravitational radiation waveforms have been performed in the PN approximation with the binary components, whether black holes or neutron stars, being treated as point masses [3 5]. These investigations are therefore appropriate in the early inspiral phase when the stars are still well separated and tidal effects can be safely neglected. Semi-analytic [6-13] and numerical [14] studies of the coalescence waveform from binary neutron stars also have been performed using Newtonian hydrodynamics and the quadrupole radiation approximation. These studies treat Newtonian effects arising from the finite size of the interacting fluid stars and focus on their tidal distortion, disruption, and eventual merger. Numerical simulations of coalescing black hole and neutron star binaries are also underway in

full general relativity (eg. 15,16]). Complicated by the challenges of treating both matter and strong gravitational fields in $(3+1)$ dimensional spacetime, these simulations are essential for obtaining definitive, quantitative results for the highly relativistic interactions which generate the late inspiral and final coalescence waveforms.

There is an important distinction between binaries in Newtonian theory and those in general relativity: In Newtonian theory a binary can exist in true dynamic equilibrium, but in general relativity true equilibrium cannot exist because of the generation of gravitational 
waves. For binaries with sufficiently large orbital separation $(r>>M)$, the orbital decay time due to emission of gravitational waves is much longer than the orbital timescale, so that the inspiral is quasi-static through a sequence of quasi-equilibrium configurations. The inspiral becomes dynamic and quasi-equilibrium is destroyed with the onset of relativistic orbital instabilities $(r \sim$ few $M)$. Dynamic behavior may also commence when the purely Newtonian tidal instability is reached near $r \sim$ few $R$ (eg., 8 11,14). For these reasons, both Newtonian and relativistic effects are important.

In the relativistic simulations of Wilson, Mathews, and Marronetti [16], the timedependent equations of relativistic hydrodynamics are solved in a simplified, strong-field background, and the system is relaxed to find quasi-static equilibrium configurations of corotating neutron star binaries for a specific equation of state. These authors claim that neutron stars just below the maximum (isolated) mass become unstable and collapse to black holes when placed in close binary orbits, in contrast to the Newtonian result that the tidal field tends to stabilize a star (see Fig. 15 of Ref. [8]; also Ref. [17]). Such collapse could significantly affect the inspiral and the associated gravitational waveform, and therefore deserves careful attention.

Recently, Baumgarte et al. [18] have constructed quasi-static binary equilibrium models in full general relativity. However, their numerical models, based on a polytropic equation of state, are restricted to be corotating. This work complements the numerical analysis of Cook [19], who analyzed binary black holes with zero spins.

A fully general relativistic calculation of a binary neutron star configuration is a computationally intensive problem. By contrast, an ellipsoidal figure of equilibrium (EFE) treatment, while only approximate, can find an equilibrium configuration in less than a second on a typical workstation. This speed affords a quick means of gaining a qualitative understanding of the stellar models and equilibrium sequence. These sequences can help provide a better understanding of the general relativistic solutions which are now being generated (eg. Ref. [18]). In addition, while general relativistic codes are currently limited to corotation, or near corotation, an EFE treatment allows straightforward construction and comparison of 
both corotating and irrotational sequences. The second order variation of the functional can be used to identify instabilities, e.g. the innermost stable circular orbit (ISCO) or the collapse of a massive neutron star in a binary to a black hole. Furthermore, both secular and dynamical instabilities can be identified and distinguished.

Taniguchi and Nakamura [20] use the EFE approach to model black hole-neutron star binaries in the Roche approximation, using a pseudo-potential to model the strong-field orbital motion. Lai and Wiseman [21] insert EFE terms in the hybrid- $\mathrm{P}^{2} \mathrm{~N}$ dynamical equations of motion, which are then integrated to identify the ISCO. The EFE approach has also recently been extended to first PN order in treatments of corotating binaries [22].

In this paper, we adopt the EFE energy variational method, extended to first PN order. Our energy functional includes terms for both the internal hydrodynamic structure as well as the external orbital motion. We add the leading PN correction to the internal and selfgravitational energy of the stars. We adopt the hybrid expressions of [4], to PN order, to give the orbital terms; these expressions are exact in the test mass limit and correct to PN order for arbitrary masses. The energy functional is varied to find quasi-equilibrium configurations of both corotating and irrotational binary sequences, parameterized by orbital separation (or orbital angular velocity).

In $\S[$ we outline the basic approximations of our EFE scheme. In $\S I I I$ we give the various energy, angular momentum, and circulation terms which comprise our functionals. The method of solution is discussed in $\S \mathrm{IV}$, and results are presented in $\S \mathrm{V}$. Finally, we discuss the implications of our results and directions for future work in $\S \mathrm{VI}$. In the appendices, we derive the PN energy contributions for an isolated spherical star obeying a polytropic equation of state.

\section{FUNDAMENTAL APPROXIMATIONS}

In this section, we describe the fundamental approximations and assumptions behind our energy variational method, which we apply to construct general Darwin-Riemann equi- 
librium models. Further details and applications of our method can be found in 8 11].

In steady state, an isolated, self-gravitating binary system is characterized by conserved global quantities such as the rest (baryonic) mass $M_{o}$ and $M_{o}^{\prime}$ of each star and the total angular momentum $J$. The total energy of the system can be written as a functional of the rest mass density and velocity distributions $\rho_{o}(\mathbf{x})$ and $\mathbf{v}(\mathbf{x})$, respectively, even if the system is not in equilibrium. In principle, an equilibrium configuration can be determined by extremizing this energy functional with respect to all variations of $\rho_{o}(\mathbf{x})$ and $\mathbf{v}(\mathbf{x})$ that leave the conserved quantities unchanged. The fundamental approximation of our method is to replace the infinite number of degrees of freedom contained in $\rho_{o}(\mathbf{x})$ and $\mathbf{v}(\mathbf{x})$ by a finite number of parameters $\gamma_{1}, \gamma_{2}, \ldots$, so that the total energy is a function only of these parameters:

$$
E=E\left(\gamma_{1}, \gamma_{2}, \ldots ; M_{o}, M_{o}^{\prime}, J, \ldots\right)
$$

An equilibrium configuration is then determined by extremizing the energy according to

$$
\frac{\partial E}{\partial \gamma_{i}}=0, \quad i=1,2, \ldots
$$

where the partial derivatives are taken holding the conserved quantities $M_{o}, M_{o}^{\prime}, J, \ldots$ constant.

An expression like equation (11) can be written down only with the help of simplifying assumptions. We adopt the compressible ellipsoidal approximation to model the neutron stars, i.e. we assume they are triaxial ellipsoids supported in hydrostatic equilibrium by a polytropic equation of state [6, $8,12,23]$. Surfaces of constant density are assumed to be concentric self-similar ellipsoids. These approximations become exact in the limit that the fluid is incompressible and the gravitational potential is strictly Newtonian and truncated beyond the tidal term [23]. For compressible configurations, this simplification is equivalent to using an ellipsoidal trial function in an energy functional and then extremizing the functional to find the equilibrium solution. Comparisons with numerical simulations show that the ellipsoidal model is reliable, particularly for stars obeying stiff equations of state [8 11]. 
The velocity field of the fluid is taken to be that of a Riemann-S ellipsoid, in which all internal velocities are linear functions of the coordinates (see eg. Ref. [8], §5, for details). The vorticity vector is assumed to be everywhere parallel to the orbital rotation axis. Since the viscosity in neutron stars is thought to be negligible, the two stars will conserve circulation as they inspiral [6.77, so that slowly spinning neutron stars at $r=\infty$ will maintain a small, constant circulation. However, current general relativistic quasi-equilibrium codes are forced to consider corotating, or nearly corotating, sequences rather than sequences of constant circulation. We therefore concentrate on two interesting limiting cases: irrotational sequences (for non-synchronized systems with zero circulation) and corotating sequences (corresponding to synchronized binary systems in uniform rotation).

Consider a binary system composed of two stars of rest mass $M_{o}$ and $M_{o}^{\prime}$ in circular orbit. Throughout this paper unprimed quantities refer to the star of rest mass $M_{o}$ while primed quantities refer to the star of mass $M_{o}^{\prime}$. Following Ref. [23], we denote the mass ratio as $p \equiv M_{o} / M_{o}^{\prime}$. The density and pressure are related by

$$
P=K \rho_{o}^{(1+1 / n)}, \quad P^{\prime}=K^{\prime} \rho_{o}^{\prime\left(1+1 / n^{\prime}\right)} .
$$

Note that our treatment allows the two stars to have both distinct polytropic indices $\left(n \neq n^{\prime}\right)$ and polytropic constants $\left(K \neq K^{\prime}\right)$, although in this paper we concentrate on the case of two identical stars. The binary separation is denoted by $r$, and the principal axes of the two ellipsoids by $a_{1}, a_{2}, a_{3}$, and $a_{1}^{\prime}, a_{2}^{\prime}, a_{3}^{\prime}$. The orientation is such that $a_{1}$ and $a_{1}^{\prime}$ are measured along the binary axis, $a_{2}$ and $a_{2}^{\prime}$ in the direction of the orbital motion, and $a_{3}$ and $a_{3}^{\prime}$ along the rotation axis. In place of the three principal axes $a_{i}$, it is often convenient to introduce as independent variables the central rest mass density $\rho_{o c}$, and two oblateness parameters defined as

$$
\lambda_{1} \equiv\left(\frac{a_{3}}{a_{1}}\right)^{2 / 3}, \quad \lambda_{2} \equiv\left(\frac{a_{3}}{a_{2}}\right)^{2 / 3}
$$

Similarly we can introduce $\rho_{o c}^{\prime}, \lambda_{1}^{\prime}$, and $\lambda_{2}^{\prime}$ in place of the three $a_{i}^{\prime}$. Thus the seven independent variables which parameterize our models are $\left\{r, \rho_{o c}, \lambda_{1}, \lambda_{2}, \rho_{o c}^{\prime}, \lambda_{1}^{\prime}, \lambda_{2}^{\prime}\right\}$. 
As is standard in EFE treatments, we assume that the density profile $\rho(m)$, where $m$ is the mass interior to an isodensity surface, is identical to that of a spherical polytrope with the same $K$ and $n$, but with radius $R=\left(a_{1} a_{2} a_{3}\right)^{1 / 3}$ [see eq. (B19)]. In particular, to PN order, a star with semi-major axes $a_{i}$ has the same central density as a spherical star (not necessarily in equilibrium) with radius $R$ in Schwarzschild coordinates,

$$
\rho_{o c}=\frac{M_{o} \xi_{1}}{4 \pi R^{3}\left|\theta_{1}^{\prime}\right|}\left\{1+\frac{G M_{o}}{R c^{2}} \frac{1}{n+1}\left[\frac{3 \alpha_{1}}{\xi_{1}^{2}\left|\theta_{1}^{\prime}\right|^{2}}+\frac{\beta_{1}}{\xi_{1}\left|\theta_{1}^{\prime}\right|^{2}}+\frac{(n-3)(n+1)}{5-n}\right]\right\},
$$

where we have used equations (A14) and (B37). Here $\theta$ and $\xi$ are the usual Lane-Emden variables for a polytrope (see, e.g., Ref. [24]) with $\theta\left(\xi_{1}\right)=0$, and where the quantities $\alpha_{1}$ and $\beta_{1}$ are defined in Appendix $\mathrm{A}$ and depend only on the polytropic index $n$ (for $n=0.5$ we have $\alpha_{1}=-3.39307$ and $\beta_{1}=3.63708$, while for $n=1.0$ we have $\alpha_{1}=-2.77960$ and $\left.\beta_{1}=2.67168\right)$. In the Newtonian limit $(c \rightarrow \infty)$, equation (5) reduces to the expression

used in the Newtonian treatment of 8 11]. Although equation (5) originates from a PN expansion, our code treats this relation as exact.

\section{ENERGY, ANGULAR MOMENTUM, AND CIRCULATION TERMS}

\section{A. Newtonian Treatment}

We now review the energy, angular momentum, and circulation terms in the Newtonian EFE approximation, which has been covered in detail in [8 [1]. When similar expressions can be written for the two stars, we give only the one corresponding to $M_{o}$, the other being obtained simply by replacing unprimed with primed quantities.

The usual Newtonian orbital contributions to the energy and angular momentum are

$$
E_{N \text { orb }}=\frac{1}{2} \mu \Omega^{2} r^{2}-\frac{G M_{o} M_{o}^{\prime}}{r}, \quad J_{N \text { orb }}=\mu r^{2} \Omega
$$

where $\mu=M_{o} M_{o}^{\prime} /\left(M_{o}+M_{o}^{\prime}\right)$ is the reduced mass and $\Omega=v / r$ is the orbital angular velocity. The gravitational tidal (quadrupole) interaction energy $W_{t}$ between the two stars is (see Appendix B of Ref. 88) 


$$
W_{t}=-\frac{G M_{o}}{2 r^{3}}\left(2 I_{11}^{\prime}-I_{22}^{\prime}-I_{33}^{\prime}\right)-\frac{G M_{o}^{\prime}}{2 r^{3}}\left(2 I_{11}-I_{22}-I_{33}\right)
$$

Here we have defined

$$
I_{j j}=\frac{h_{j}\left(\lambda_{1}, \lambda_{2}\right)}{4 k_{3}} M_{o}^{5 / 3} \rho_{o c}^{-2 / 3} \quad(\text { no summation })
$$

where

$$
h_{1} \equiv \frac{\lambda_{2}}{\lambda_{1}^{2}}, \quad h_{2} \equiv \frac{\lambda_{1}}{\lambda_{2}^{2}}, \quad h_{3} \equiv \lambda_{1} \lambda_{2}
$$

with analogous relations for $I_{j j}^{\prime}$ and $h_{j}^{\prime}$. We will refer to key relations in Ref. [8] by their equation number preceded by an "I"; for example, equations (8) and (9) are also equations (I.B8) and (I.B9), respectively. The dimensionless structure coefficient $k_{3}$ appearing in equation (8) is defined by [cf. eq. (I.3.17)]

$$
k_{3}=\frac{5}{4 \kappa_{n}}\left(\frac{4 \pi\left|\theta_{1}^{\prime}\right|}{\xi_{1}}\right)^{2 / 3}
$$

where

$$
\kappa_{n} \equiv \frac{5}{3} \frac{\int_{0}^{\xi_{1}} \theta^{n} \xi^{4} d \xi}{\xi_{1}^{4}\left|\theta^{\prime}{ }_{1}\right|}
$$

so that $\kappa_{n}=1$ for $n=0$. Values of $k_{3}$ and $\kappa_{n}$ for various $n$ are tabulated in Table I of [8].

The internal energy of the star with mass $M_{o}$ is given simply by

$$
U=k_{1} K \rho_{o c}^{1 / n} M_{o}
$$

The self-gravitational energy can be written [cf. eq. (I.4.6)]

$$
W=-k_{2} G M_{o}^{5 / 3} \rho_{o c}^{1 / 3} f
$$

where the dimensionless ratio $f$ is given by

$$
f=f\left(\lambda_{1}, \lambda_{2}\right) \equiv \frac{A_{1} a_{1}^{2}+A_{2} a_{2}^{2}+A_{3} a_{3}^{2}}{2\left(a_{1} a_{2} a_{3}\right)^{2 / 3}}
$$

so that $f=1$ for a spherical star. The index symbols $A_{i}$ are defined as in Ref. [23] ( $\left.\$ 17\right)$, 


$$
A_{i} \equiv a_{1} a_{2} a_{3} \int_{0}^{\infty} \frac{d u}{\Delta\left(a_{i}^{2}+u\right)}, \quad \text { with } \quad \Delta^{2}=\left(a_{1}^{2}+u\right)\left(a_{2}^{2}+u\right)\left(a_{3}^{2}+u\right)
$$

and are functions only of $\lambda_{1}$ and $\lambda_{2}$. In equations (12) and (13), $k_{1}$ and $k_{2}$ are dimensionless structure constants depending only on the polytropic index $n$, defined as

$$
k_{1} \equiv \frac{n(n+1)}{5-n} \xi_{1}\left|\theta^{\prime}{ }_{1}\right|, \quad k_{2} \equiv \frac{3}{5-n}\left(\frac{4 \pi\left|\theta^{\prime}{ }_{1}\right|}{\xi_{1}}\right)^{1 / 3}
$$

Values of $k_{1}$ and $k_{2}$ are tabulated in Ref. [8].

We permit the star to be differentially rotating with uniform vorticity $\zeta \mathbf{e}_{\mathbf{3}}$ as measured in the corotating frame of the binary:

$$
\zeta \equiv(\nabla \times \mathbf{u}) \cdot \mathbf{e}_{\mathbf{3}}=-\frac{a_{1}^{2}+a_{2}^{2}}{a_{1} a_{2}} \Lambda .
$$

Here the velocity field $\mathbf{u}$ in the corotating frame is given by

$$
\mathbf{u}=Q_{1} x_{2} \mathbf{e}_{1}+Q_{2} x_{1} \mathbf{e}_{2}
$$

with

$$
\begin{aligned}
& Q_{1}=-\frac{a_{1}^{2}}{a_{1}^{2}+a_{2}^{2}} \zeta=+\frac{a_{1}}{a_{2}} \Lambda, \\
& Q_{2}=+\frac{a_{2}^{2}}{a_{1}^{2}+a_{2}^{2}} \zeta=-\frac{a_{2}}{a_{1}} \Lambda .
\end{aligned}
$$

The quantity $\Lambda$ is the angular velocity of the internal fluid motions in the corotating frame. Here $\mathbf{e}_{\mathbf{1}}$ is along the binary axis, directed from $M_{o}$ to $M_{o}^{\prime}, \mathbf{e}_{\mathbf{2}}$ is in the direction of the orbital velocity, and $\mathbf{e}_{\mathbf{3}}$ is perpendicular to the orbital plane. The velocity field in the inertial frame is given by

$$
\mathbf{u}^{(0)}=\mathbf{u}+\Omega \times \mathbf{x},
$$

and the vorticity in this frame is

$$
\zeta^{(0)}=\left(\nabla \times \mathbf{u}^{(0)}\right) \cdot \mathbf{e}_{3}=\left(2+f_{R}\right) \Omega,
$$

where 


$$
f_{R}=\frac{\zeta}{\Omega}
$$

Corotation (synchronization) corresponds to $f_{R}=\zeta=\Lambda=0$, while an irrotational velocity field is obtained when $f_{R}=-2$. Note that the geometric outer shapes of the two stars always rotate at the orbital angular velocity $\Omega$ in the inertial frame, regardless of the internal velocity field.

The Newtonian spin kinetic energy $T_{s}$ (i.e., the kinetic energy in internal fluid motions) in the inertial frame can be obtained from equations (18)-(20). Assuming a Lane-Emden trial density function, one finds [cf. eq. (I.5.6)]

$$
T_{s}=\frac{1}{2} I\left(\Lambda^{2}+\Omega^{2}\right)-2\left(I_{11} I_{22}\right)^{1 / 2} \Lambda \Omega
$$

where $I=I_{11}+I_{22}$ is the moment of inertia. Similarly, the Newtonian spin angular momentum $J_{s}$ can be written [cf. eq. (I.5.5)]

$$
J_{s}=I \Omega-2\left(I_{11} I_{22}\right)^{1 / 2} \Lambda
$$

As given by [8 11], the purely Newtonian total energy of the system, not necessary in equilibrium, is therefore

$$
E_{N}=E_{N o r b}+U+U^{\prime}+W+W^{\prime}+T_{s}+T_{s}^{\prime}+W_{t}
$$

Similarly, the total angular momentum to Newtonian order is

$$
J_{N}=J_{N \text { orb }}+J_{s}+J_{s}^{\prime}
$$

Another important quantity, conserved in the absence of viscosity, is the fluid circulation $C$ along the equator of the star (see $\S 5.1$ of Ref. [8]). We define

$$
\mathcal{C} \equiv\left(-\frac{1}{5 \pi} \kappa_{n} M_{o}\right) C=I \Lambda-2\left(I_{11} I_{22}\right)^{1 / 2} \Omega
$$

The quantity $\mathcal{C}$ has the dimensions of angular momentum but is proportional to the conserved circulation $C$. For convenience, we typically refer to $\mathcal{C}$ itself as the circulation. 


\section{B. Post-Newtonian Treatment}

In this paper, we augment the Newtonian terms of $\S \amalg I \mathrm{~A}$ with PN contributions (a) to the orbital energy and angular momentum, (b) to the internal and self-gravitational energy of a star, and (c) to the coupling energy and angular momentum between the internal structure and the orbital motion.

We replace of the Newtonian contributions to orbital energy and angular momentum [eq. (6)] with the hybrid-PN expressions of [4] (with $\dot{r}=0$, valid for circular orbits):

$$
E_{H}=E_{T}+E_{\eta}, \quad J_{H}=J_{T}+J_{\eta}
$$

where the Schwarzschild, or test-mass, contributions are (with $G=c=1$ for these two equations only)

$$
\begin{aligned}
& E_{T}=\mu\left\{\left(\frac{r-m}{r+m}\right)^{1 / 2}\left[1-v^{2} \frac{(r+m)^{3}}{r^{2}(r-m)}\right]^{-1 / 2}-1\right\}, \\
& J_{T}=\mu v r\left\{\left[\frac{(r+m)^{5}}{r^{4}(r-m)}\right]^{1 / 2}\left[1-v^{2} \frac{(r+m)^{3}}{r^{2}(r-m)}\right]^{-1 / 2}\right\},
\end{aligned}
$$

and the PN corrections unaccounted for by the Schwarzschild terms are

$$
\begin{aligned}
& E_{\eta}=-\eta \mu v^{2}\left(\frac{9}{8} \frac{v^{2}}{c^{2}}-\frac{1}{2} \frac{G m}{r c^{2}}\right), \\
& J_{\eta}=-\eta \mu v r\left(\frac{3}{2} \frac{v^{2}}{c^{2}}-\frac{G m}{r c^{2}}\right) .
\end{aligned}
$$

Here $v=\Omega r$ is the orbital velocity, the total rest mass $m=M_{o}+M_{o}^{\prime}$, and the mass parameter $\eta \equiv \mu / m$. In the notation of Ref. [4], $m$ also includes contributions from the self energy of the stars and is not simply the total rest mass; however, we account for these contributions below [see eqs. (35) and (36)] as a coupling between the internal structure and the orbital motion. With our definitions, the quantities $m$ and $\eta$ remain strictly constant along the sequence. The radius $r$ is the so-called harmonic or de Donder coordinate. When expanded in $G m / r c^{2}$, the above expressions for $E_{H}$ and $J_{H}$ give the Newtonian terms [eq. (66)] to lowest order and the correct orbital PN terms to first order. 
The PN contributions to the internal and self-gravitational energy of a spherical polytrope are derived in Appendix B:

$$
\begin{aligned}
U_{P N} & =-l_{1} \frac{G}{c^{2}} K \rho_{o c}^{\frac{1}{n}+\frac{1}{3}} M_{o}^{5 / 3}, \\
W_{P N} & =-l_{2} \frac{G^{2}}{c^{2}} M_{o}^{7 / 3} \rho_{o c}^{2 / 3}
\end{aligned}
$$

where the coefficients $l_{1}$ and $l_{2}$ are defined by equations (B42) and (B52), respectively, and depend only on the polytropic index $n$. Values for $l_{1}$ and $l_{2}$ are listed in Table [- We also include the analogous expressions for the PN energy contributions $U_{P N}^{\prime}$ and $W_{P N}^{\prime}$ in the second star. For isolated stars, variation of the energy function $E\left(\rho_{o c}\right)=U+W+U_{P N}+W_{P N}$ does a good job of approximating the exact equilibrium sequence obtained by integrating the Oppenheimer-Volkoff (OV) equation, especially for soft equations of state (see Figure (1)).

We neglect PN corrections due to asphericity; for small perturbations from spherical, equations (33) and (34) are valid to lowest order. To account completely for all PN corrections to the internal and self-gravitational energy, equations (33) and (34) would need to be multiplied by a form factor analogous to $f\left(\lambda_{1}, \lambda_{2}\right)$ in equation (14). We also neglect $\mathrm{P}^{2} \mathrm{~N}$ and higher order corrections to the internal and self-gravitational energy of the neutron stars.

The final PN correction terms we include involve the coupling between the orbital motion and the internal structure. Effacement of internal structure (see Ref. [25] and references therein) requires that we substitute the effective masses $M_{\text {eff }}=M_{o}+E_{\text {self }} / c^{2}$ and $M_{\text {eff }}^{\prime}=$ $M_{o}^{\prime}+E_{\text {self }}^{\prime} / c^{2}$ in place of the rest masses $M_{o}$ and $M_{o}^{\prime}$ in the Newtonian equation (6) if we wish to maintain PN order accuracy. Here the self energy of the mass $M_{o}$ neutron star is $E_{\text {self }}=W+U+U_{P N}+W_{P N}$. We have chosen to exclude the spin kinetic energy $T_{s}$ from the self energy $E_{\text {self }}$ in order to ensure that our binary configurations satisfy the identity $d E=\Omega d J$ exactly (see $\oint[\bar{\square})$. We speculate that additional higher order terms which we are neglecting would restore this identity when we include $T_{s}$. Furthermore, $T_{s}$ is typically small, especially for irrotational sequences, so that we are neglecting a term which is smaller than our order of accuracy. Expanding to PN order, the resulting corrections to the Newtonian 
point-mass energy and angular momentum are

$$
\begin{aligned}
E_{O I} & =\frac{E_{\text {self }}}{c^{2}}\left(\frac{1}{2} \frac{M_{o}^{\prime 2}}{m^{2}} \frac{v^{2}}{c^{2}}-\frac{G M_{o}^{\prime}}{r}\right), \\
J_{O I} & =\frac{E_{\text {self }}}{c^{2}} \frac{M_{o}^{\prime 2}}{m^{2}} v r
\end{aligned}
$$

with analogous expressions $E_{O I}^{\prime}$ and $J_{O I}^{\prime}$ due to the corrections arising from the self energy of the neutron star of mass $M_{o}^{\prime}$. The subscript OI serves as a reminder that these terms couple the orbital motion to the internal structure of the neutron stars. Although including $U_{P N}$ and $W_{P N}$ as part of $E_{\text {self }}$ in equations (35) and (36) amounts to keeping terms which are formally of $\mathrm{P}^{2} \mathrm{~N}$ order, doing so keeps our energy functional accurate even for stars at $r=\infty$ but with $v>0$; excluding $U_{P N}$ and $W_{P N}$ from $E_{\text {self }}$ would lead to an energy functional which yields manifestly incorrect results for isolated stars (in particular, the stability of a star would depend on the velocity of the observer).

We neglect PN effects in the internal motion which would appear as corrections to the spin kinetic energy $T_{s}$, the spin angular momentum $J_{s}$, and the circulation $C$. For corotating sequences, these corrections are on the order of $R / r$ times the PN orbital terms, so that we are justified in neglecting PN internal motion effects all the way down to roughly the ISCO $(r \sim$ few $R$ ). For irrotational sequences, PN internal motion is especially small, since the fluid motion in the inertial frame is always predominately set by the orbital motion. We are also neglecting all PN tidal terms (but see $\S \mathrm{VIC}$ ).

The total energy and angular momentum functionals used in our treatment are therefore $E=E_{H}+U+U^{\prime}+W+W^{\prime}+T_{s}+T_{s}^{\prime}+W_{t}+U_{P N}+U_{P N}^{\prime}+W_{P N}+W_{P N}^{\prime}+E_{O I}+E_{O I}^{\prime}$,

and

$$
J=J_{H}+J_{s}+J_{s}^{\prime}+J_{O I}+J_{O I}^{\prime}
$$

Since we are approximating the internal fluid motion as Newtonian, we continue to use equation (27) for the circulation. 


\section{FINDING EQUILIBRIUM SEQUENCES}

In order to find an equilibrium solution, we must extremize the energy function $E$ while holding all the quantities $M_{o}, M_{o}^{\prime}, \mathcal{C}, \mathcal{C}^{\prime}$, and $J$ constant. We are therefore identifying those configurations for which the energy is unchanged to first order by any perturbation which conserves rest mass, circulation, and total angular momentum. An equilibrium sequence is constructed by repeating this extremization at various values of the orbital separation $r$. In the case of corotation, the stars are members of the Riemann-S family with vorticity $\zeta=0$, and although the perturbations considered conserve circulation, the circulation does indeed vary along the equilibrium sequence itself [8, 11].

Unfortunately, the various energy terms in $\$$ [II do not depend explicitly on $J, \mathcal{C}$, and $\mathcal{C}^{\prime}$. Instead, the energy has been written in terms of the variables $\sigma_{i}$ from the set $\left\{r, \rho_{o c}, \lambda_{1}, \lambda_{2}, \rho_{o c}^{\prime}, \lambda_{1}^{\prime}, \lambda_{2}^{\prime}, \Omega, \Lambda, \Lambda^{\prime}\right\}$, and these variables are not independent. The Lagrange multiplier method allows us straightforwardly to minimize the total energy $E\left(\sigma_{i}\right)$ subject to the constraints that the total angular momentum $J\left(\sigma_{i}\right)$ and the circulations $\mathcal{C}\left(\sigma_{i}\right)$ and $\mathcal{C}^{\prime}\left(\sigma_{i}\right)$ are constant. The equations which determine an equilibrium configuration are

$$
\frac{\partial E}{\partial \sigma_{i}}+\xi_{J} \frac{\partial J}{\partial \sigma_{i}}+\xi_{\mathcal{C}} \frac{\partial \mathcal{C}}{\partial \sigma_{i}}+\xi_{\mathcal{C}^{\prime}} \frac{\partial \mathcal{C}^{\prime}}{\partial \sigma_{i}}=0
$$

where $\xi_{J}, \xi_{\mathcal{C}}$, and $\xi_{\mathcal{C}^{\prime}}$ are the Lagrange multipliers. Here the partial differentiation with respect to $\sigma_{i}$ is done with all the remaining variables $\sigma_{j}(j \neq i)$ being held constant. Although equations (39) are accurate only to the order of our expansion of $E, J, \mathcal{C}$, and $\mathcal{C}^{\prime}$, our numerical code treats them as exact. We expanded and restructured a version of the code developed in Ref. [11] to solve these coupled equations numerically. For a given value of $r$, we use a Newton-Raphson scheme to solve the 10 equations (39) and find the variables $\rho_{\text {oc }}, \lambda_{1}, \lambda_{2}, \rho_{o c}^{\prime}, \lambda_{1}^{\prime}, \lambda_{2}^{\prime}, \Omega, \xi_{J}, \xi_{\mathcal{C}}$, and $\xi_{\mathcal{C}^{\prime}}$. In practice, our code uses Cramer's rule on the $\sigma_{i} \in$ $\left\{\Omega, \Lambda, \Lambda^{\prime}\right\}$ equations of (39) to find the Lagrange multipliers: 


$$
\xi_{J}=\frac{-1}{d}\left|\begin{array}{ccc}
\frac{\partial E}{\partial \Omega} & \frac{\partial \mathcal{C}}{\partial \Omega} & \frac{\partial \mathcal{C}^{\prime}}{\partial \Omega} \\
\frac{\partial E}{\partial \Lambda} & \frac{\partial \mathcal{C}}{\partial \Lambda} & \frac{\partial \mathcal{C}^{\prime}}{\partial \Lambda} \\
\frac{\partial E}{\partial \Lambda^{\prime}} & \frac{\partial \mathcal{C}}{\partial \Lambda^{\prime}} & \frac{\partial \mathcal{C}^{\prime}}{\partial \Lambda^{\prime}}
\end{array}\right|, \quad \xi_{\mathcal{C}}=\frac{-1}{d}\left|\begin{array}{ccc}
\frac{\partial J}{\partial \Omega} & \frac{\partial E}{\partial \Omega} & \frac{\partial \mathcal{C}^{\prime}}{\partial \Omega} \\
\frac{\partial J}{\partial \Lambda} & \frac{\partial E}{\partial \Lambda} & \frac{\partial \mathcal{C}^{\prime}}{\partial \Lambda} \\
\frac{\partial J}{\partial \Lambda^{\prime}} & \frac{\partial E}{\partial \Lambda^{\prime}} & \frac{\partial \mathcal{C}^{\prime}}{\partial \Lambda^{\prime}}
\end{array}\right|, \quad \xi_{\mathcal{C}^{\prime}}=\frac{-1}{d}\left|\begin{array}{ccc}
\frac{\partial J}{\partial \Omega} & \frac{\partial \mathcal{C}}{\partial \Omega} & \frac{\partial E}{\partial \Omega} \\
\frac{\partial J}{\partial \Lambda} & \frac{\partial \mathcal{C}}{\partial \Lambda} & \frac{\partial E}{\partial \Lambda} \\
\frac{\partial J}{\partial \Lambda^{\prime}} & \frac{\partial \mathcal{C}}{\partial \Lambda^{\prime}} & \frac{\partial E}{\partial \Lambda^{\prime}}
\end{array}\right|,
$$

where the determinant

$$
d=\left|\begin{array}{lll}
\frac{\partial J}{\partial \Omega} & \frac{\partial \mathcal{C}}{\partial \Omega} & \frac{\partial \mathcal{C}^{\prime}}{\partial \Omega} \\
\frac{\partial J}{\partial \Lambda} & \frac{\partial \mathcal{C}}{\partial \Lambda} & \frac{\partial \mathcal{C}^{\prime}}{\partial \Lambda} \\
\frac{\partial J}{\partial \Lambda^{\prime}} & \frac{\partial \mathcal{C}}{\partial \Lambda^{\prime}} & \frac{\partial \mathcal{C}^{\prime}}{\partial \Lambda^{\prime}}
\end{array}\right|
$$

Using equations (40), we substitute for the Lagrange multipliers in the remaining 7 equations of (39) to find the orbital velocity as well as the shape and structure of the neutron stars.

The Lagrange multipliers can even be determined analytically, since expressions for the various derivatives appearing in equations (40) and (41) can be derived from equations (37), (38), and (27). The algebra is simplified somewhat by $\partial \mathcal{C} / \partial \Lambda^{\prime}=0$ and $\partial \mathcal{C}^{\prime} / \partial \Lambda=0$. We find

$\xi_{J}=-\Omega, \xi_{\mathcal{C}}=-\Lambda$, and $\xi_{\mathcal{C}^{\prime}}=-\Lambda^{\prime}$ as mathematical identities, for any $r, \rho_{o c}, \lambda_{1}, \lambda_{2}, \rho_{o c}^{\prime}, \lambda_{1}^{\prime}, \lambda_{2}^{\prime}$ and not just along equilibrium sequences. This provides a convenient check of a large portion of the code, since the Lagrange variables are determined by evaluating the determinants in equations (40) and (41) directly at every iteration in the Newton-Raphson scheme. Furthermore, equation (39) then implies

$$
d E-\Omega d J-\Lambda d \mathcal{C}-\Lambda^{\prime} d \mathcal{C}^{\prime}=0
$$

so that $d E=\Omega d J$ along both corotating $\left(\Lambda=\Lambda^{\prime}=0\right)$ and constant circulation $(d \mathcal{C}=$ $\left.d \mathcal{C}^{\prime}=0\right)$ sequences. Consequently, the minima of $J$ and $E$ always coincide precisely on our equilibrium sequences.

\section{RESULTS}

We concentrate on binaries composed of identical stars, each with polytropic index $n=$ 0.5 or 1.0 , and with a compactness parameter $G M / R c^{2}=0.125,0.2$, or 0.25 in isolation. These values of $n$ and $G M / R c^{2}$ cover an interesting region of parameter space relevant 
to neutron stars. For the AV14+UVII equation of state of Ref. [26], stars in the mass range $0.7 M_{\odot} \lesssim M \lesssim 1.7 M_{\odot}$ have compactness parameters $0.1 \lesssim G M / R c^{2} \lesssim 0.25$ with corresponding effective polytropic indices $1 \gtrsim n \gtrsim 0.5$ (see Table 3 of Ref. [10]). For an isolated neutron star with $M=1.4 M_{\odot}$, the nine equations of state in Ref. [27] which allow this mass all yield a compactness parameter in the range $0.13 \lesssim G M / R c^{2} \lesssim 0.28$.

Some physical quantities describing our neutron stars in isolation are listed in Table 凹. These quantities can be converted to physical units once a value of the polytropic constant $K$ is chosen. For instance, for $n=0.5$ and $K=1.35 \times 10^{-10} \mathrm{~cm}^{8} / \mathrm{g}^{2} / \mathrm{sec}^{2}$, the maximum gravitational mass $M^{\max }=2.20 M_{\odot}$, so that $M=0.83,1.39$, and $1.74 M_{\odot}$ and $R=9.8,10.3$, and $10.3 \mathrm{~km}$ for $G M / R c^{2}=0.125,0.2$, and 0.25 , respectively. For $n=1$ and $K=1.20 \times 10^{5}$ $\mathrm{cm}^{5} / \mathrm{g} / \mathrm{sec}^{2}$, the maximum mass $M^{\max }=1.600 M_{\odot}$ so that $M=1.15,1.52$, and $1.599 M_{\odot}$ and $R=13.6,11.2$, and $9.4 \mathrm{~km}$ for $G M / R c^{2}=0.125,0.2$, and 0.25 , respectively. Any of our models can be scaled to a particular mass (say $1.4 M_{\odot}$ ) by an appropriate choice of the polytropic constant $K$.

Integration of the OV equation reveals that the true maximum masses of $n=0.5$ and 1.0 sequences occur at $\log K \rho_{o c} / c^{2}=-0.082$ and -0.497 , respectively, at which point $G M / R c^{2}=0.316$ and $G M / R c^{2}=0.214$. We note, however, that our $n=1.0$ isolated stars with $G M / R c^{2}=0.25$ are indeed stable in our PN-accurate model, since our energy functional [eq. ( $(\overline{\mathrm{B} 44})$ ] gives $G M / R c^{2}=0.2556$ at the maximum isolated mass (see Table [II).

When expressing our results, the orbital angular velocity, angular momentum, energy, and circulation are listed in terms of the dimensionless ratios

$$
\bar{\Omega}=\frac{\Omega}{\left(\pi G \bar{\rho}_{o}\right)^{1 / 2}}, \quad \bar{J}=\frac{J}{\left(G M_{o}^{3} R_{o}\right)^{1 / 2}}, \quad \bar{E}=\frac{E}{\left(G M_{o}^{2} / R_{o}\right)}, \quad \overline{\mathcal{C}}=\frac{\mathcal{C}}{\left(G M_{o}^{3} R_{o}\right)^{1 / 2}},
$$

where $\bar{\rho}_{o}=M_{o} /\left(4 \pi R_{o}^{3} / 3\right)$. Here $R_{o}$ is defined to be the radius that an isolated polytrope, with the same $M_{o}, K$, and $n$, would have in Newtonian theory (ie. without the $U_{P N}$ and $W_{P N}$ corrections):

$$
R_{o} \equiv \xi_{1}\left[\frac{(n+1) K}{4 \pi G}\right]^{n /(3-n)}\left(\frac{M_{o}}{4 \pi \xi_{1}^{2}\left|\theta_{1}^{\prime}\right|}\right)^{(1-n) /(3-n)} .
$$


The radius $R_{o}$ is a convenient unit of length because it is constant along equilibrium sequences.

The orbital separation $r$ is measured in harmonic coordinates while the semi-major axes $a_{i}$ are measured in Schwarzschild coordinates, which is permissible because we have two distinct small parameters in our PN expansion, $G m / r c^{2}$ and $G M / R c^{2}$. We determine when the neutron stars touch by the PN accurate relation $r+G M_{t} / c^{2}=a_{1}+a_{1}^{\prime}$, where $M_{t}=$ $2 M_{o}+E / c^{2}$ is the total mass energy of the system. Since the radial coordinate $r$ is not a gauge invariant quantity, we choose to make our plots as a function of the orbital angular frequency $f=\Omega / 2 \pi$ ( $=1 / 2$ the gravitational wave frequency). The non-dimensionalized orbital velocity $\bar{\Omega}$ can be converted to a frequency $f$ in $\mathrm{Hz}$ via the relation

$$
M_{1.4} f=1788 \mathrm{~Hz}\left(5 \frac{G M_{o}}{R_{o} c^{2}}\right)^{3 / 2} \frac{M}{M_{o}} \bar{\Omega},
$$

where $M_{1.4}=M / 1.4 M_{\odot}$. For given values of $n$ and $G M / R c^{2}$, the corresponding values of

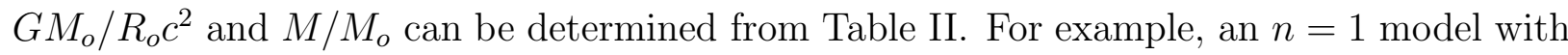
$G M / R c^{2}=0.2$ corresponds to $G M_{o} / R_{o} c^{2}=0.1467$ and $M / M_{o}=0.9083$. While $M_{o}$ and $M_{o}^{\prime}$ are constant along our equilibrium sequences, the ratio $G M / R c^{2}$ does indeed vary; however, whenever we specify a particular value of $G M / R c^{2}$ we are referring to that value at infinity (ie. for isolated neutron stars).

\section{A. Irrotational Sequences}

Figures 2 and 3 present irrotational $(\mathcal{C}=0)$ sequences in the equal mass case with $n=0.5$ and $n=1$, respectively, showing the total energy $E$, relative to its value $E_{\infty}$ at infinity, for $G M / R c^{2}=0.125,0.2$ and 0.25 (thick solid curves). The energy $E_{\infty}$ can be calculated from equation (B44) for each star. Also shown in this figure are the point-mass Newtonian equilibrium energy (thin dashed curve), as well as the results of a Newtonian EFE treatment as in [8 [1] (thick dashed line). Figure 6 shows the dependence of $G M / R c^{2}$

on the orbital frequency $f_{I S C O}$ at the ISCO for $n=0.5$ and $n=1$ irrotational sequences. 
For each $n$, the curves are terminated at the value $G M / R c^{2}$ corresponding to the maximum mass.

Table III presents physical quantities along selected irrotational sequences for $n=0.5$ and 1.0, and various values of $G M / R c^{2}$. For each sequence, the value of $r / a_{1}$ marked by a single asterisk $(*)$ identifies the equilibrium configuration for which $E$ (and $J$ ) is a minimum, ie. the ISCO. As expected, the size of the neutron star $R / R_{o}$ decreases as $G M / R c^{2}$ increases. Our $G M / R_{o} c^{2}=0$ sequences agree with the Newtonian results of Ref. [10] (see their Table $1)$.

Tables $\mathbb{I V}$ and $\mathrm{V}$ show the sizes of the individual energy and angular momentum terms appearing in equations (37) and (38) for the specific sequence $G M / R c^{2}=0.2, n=1$. Note that some of the relativistic corrections are included in $E_{H}$ and $J_{H}$ : for instance, at the ISCO, $E_{H}$ is 1.7 times smaller than what the Newtonian expression [eq.(6)] would give. The importance of the PN terms increases as $G M / R c^{2}$ increases, and the stars behave more like point masses.

\section{B. Corotational Sequences}

Fully relativistic calculations of binary neutron star systems (eg. [16,18]) provide a nice test of our PN models. However, such calculations are currently limited to corotating, or nearly corotating, stars. Figures 5 and 6 present corotating $\left(\Lambda=\Lambda^{\prime}=0\right)$ sequences with $n=0.5$ and $n=1$, respectively, showing the total energy $E$, relative to its value $E_{\infty}$ at infinity, for $G M / R c^{2}=0.125,0.2$, and 0.25 (thick solid curves).

Tables V1 presents physical quantities along selected corotating sequences. For each sequence, the value of $r / a_{1}$ marked by a single asterisk $(*)$ identifies the equilibrium configuration for which $E$ (and $J$ ) is a minimum, the innermost secularly stable circular orbit, while the value marked by a double asterisk $(* *)$ identifies the innermost dynamically stable

circular orbit. The dynamical ISCO occurs when $0=d E / d r$ at constant circulation along an equilibrium sequence (see Ref. [8]). We numerically evaluated $d E / d r$ simply by finding 
equilibrium configurations at two slightly different orbital separations and then finite differencing. As in our irrotational sequences, the overall size of the neutron star $R / R_{o}$ does indeed decrease as $G M / R c^{2}$ is increased. For corotation, the spin of each star causes a rotational bulge such that $a_{2}>a_{3}$. The circulation $\mathcal{C}$ grows in magnitude as the radial separation of the neutron stars decreases.

\section{Innermost Stable Circular Orbit}

Table VII presents the total angular momentum $J$, the total mass energy $M_{t}=2 M_{o}+$ $E / c^{2}, a_{2} / a_{1}, a_{3} / a_{1}$ and the orbital frequency $f$, all at the ISCO of selected irrotational sequences. For any given $n$, the orbital frequency $f$ can be converted into units of $\mathrm{Hz}$ if either the gravitational mass $M$ or the equation of state (ie. the polytropic constant $K$ ) is specified. It is therefore useful to express the ISCO frequency both as $M_{1.4} f_{I S C O}[\mathrm{~Hz}]$ (when $M$ is known) and $M_{1.6}^{\max } f_{I S C O}[\mathrm{~Hz}]$ (when the equation of state is known), where $M_{1.4}=M /\left(1.4 M_{\odot}\right)$ and $M_{1.6}^{\max }=M^{\max } /\left(1.6 M_{\odot}\right)$. Here $M^{\max }$ is the maximum gravitational mass in isolation for a given equation of state. The conversion factor between $M_{1.4} f_{I S C O}$ and $M_{1.6}^{\max } f_{I S C O}$ depends on the quantity $M / M^{\max }$, which can be determined from the last two columns of Table II.

Table VIII lists the orbital frequency $f_{I S C O}^{\mathrm{sec}}$ at the innermost secularly stable orbit as well

as the frequency $f_{I S C O}^{\mathrm{dyn}}$ at the innermost dynamically stable orbit. For the six combinations of $n$ and $G M / R c^{2}$ listed in this table, the frequency $f_{I S C O}^{\text {dyn }}$ differs by less than $4 \%$ from the $f_{I S C O}$ values of the corresponding irrotational sequences. That the spin has only a small effect on the orbital dynamics is consistent with the small values of $T_{s} /|W|$ (typically on the order of 0.01 near the ISCO) listed in Table $\mathrm{VI}$.

\section{Maximum mass}

An important issue is whether the maximum mass of each neutron star increases or decreases as the binary components are brought together. Figure 7 shows the rest mass 
$M_{o}$, normalized to the maximum rest mass $M_{o}^{\infty, \max }$ at infinite separation, as a function of the central density parameter $K \rho_{o c}^{1 / n} / c^{2}$ for an $n=1$ corotating binary system and at three different values of the orbital angular frequency: $f=0$ (solid curve) corresponds to spherical isolated stars, $M_{1.6}^{\max } f=434 \mathrm{~Hz}$ (long dashed curve) corresponds to the innermost dynamically stable orbit for a star with $M_{o} / M_{o}^{\infty, \max }=0.938\left(G M / R c^{2}=0.2\right.$ at infinity), and $M_{1.6}^{\max } f=463 \mathrm{~Hz}$ (short dashed curve) corresponds to the innermost dynamically stable orbit for a star with $M_{o} / M_{o}^{\infty, \max }=1\left(G M / R c^{2}=0.2556\right.$ at infinity $)$. As they inspiral, stars which begin on the stable side $\left(d M_{o} / d \rho_{o c}>0\right)$ of the $f=0$ curve move along horizontal lines (since $M_{o}$ is conserved) to the left on this plot.

Figure 8 shows the maximum equilibrium rest mass $M_{o}^{\max }$ as a function of the orbital angular frequency for corotating (dashed curves) and irrotational (solid curves) sequences. For example, the maxima of the three curves in Fig. 7 gives three data points for the $n=1.0$ corotating curve in Figure 8. The curves are terminated at the values of $f$ for which stars of rest mass $M_{o}=M_{o}^{\infty, \max }$ acquire a dynamically unstable orbit. We see that the maximum equilibrium mass increases as the orbit decays, regardless of the sequence considered. We conclude that if the maximum equilibrium mass does ever decrease in a binary system, it can do so only through PN tidal terms, or higher order PN terms, which we are neglecting in this paper (see $\$ \mathrm{VIC}$ ). Note that we have not determined from our equilibrium analysis, which of our equilibria are stable. The exception is the $f=0$ (infinite separation) sequence, for which the turning point on the $M$ versus $\rho_{o c}$ plot marks the onset of radial instability to collapse.

\section{DISCUSSION}

\section{A. Summary of Results}

We have extended the work of previous studies in a number of ways. We have improved upon purely Newtonian EFE treatments by including PN effects, and improved upon point- 
mass PN treatments by including both Newtonian and PN finite-size effects. The main message of Appendix B is that equation (B44) is the PN accurate energy functional of an isolated non-rotating star of arbitrary polytropic index $n$. Nowhere in the derivation of equation (B44) is equilibrium assumed, but in equilibrium the trial density function we implement agrees to PN order with the density profile obtained by integrating the OV equation. The PN coefficients $l_{1}$ and $l_{2}$ appearing in equation (B44) are listed for various polytropic indices $n$ in Table [. Figure 1 demonstrates that using equation (B44) yields reliable results (compare the long dashed curve to the OV results given by the solid curve), especially for soft equations of state (which have a maximum mass at small $\rho_{o c}$ ). One particularly nice feature of our energy functional is that it correctly exhibits a maximum mass even for stiff equations of state. Various physical quantities for our isolated spherical models are listed in Table $[1]$.

Figures 2 and 3 , present the equilibrium energy $E$ as a function of the orbital frequency $f$ along irrotational sequences with various values of the polytropic index $n$ and compactness parameters $G M / R c^{2}$. Note that for a given $M$ and $G M / R c^{2}, n=1$ polytropes are more centrally concentrated than $n=0.5$ polytropes, so that the former behave more like point masses (ie. Newtonian effects which destabilize the orbit are less important for $n=1$ ) and have a slightly larger $M_{1.4} f_{I S C O}$. This same tendency can be seen by comparing the $n=0.5$ and $n=1.0$ corotating sequences presented in Figures 5 and 6, respectively. In these plots the minimum of the equilibrium energy $E$ marks the onset of an orbital secular instability. The dynamical instability sets in at higher orbital frequency, as can be seen from Table VI or VIII.

Tables [II and $\nabla 1$ present various quantities along selected sequences for comparisons with future studies. The Tables $\mathbb{\nabla}$ and $\nabla$ present the relatives sizes of the terms which make up our energy and angular momentum functionals; these tables allow us to explicitly examine the relative importance of the various effects which determine the orbital dynamics and the interior structure. Comparing the magnitudes of $W_{t}$ and $E_{O I}$, it is clear that both tidal and relativistic terms play an important role. Tables VII and VIII summarize 
some of the important quantities at the ISCO of our irrotational and corotating sequences, respectively. Note that for fixed $n, M_{1.4} f_{I S C O}$ always increases with $G M / R c^{2}$, since more compact stars behave more like point masses.

Although real neutron star sequences are probably close to irrotational, fully relativistic calculations of binary systems are currently limited to corotating sequences. By looking for turning points in the equilibrium energy, it is straightforward to determine the secular ISCO frequency $f_{I S C O}^{s e c}$ in such corotating sequences. It is often assumed that the actual ISCO frequency $f_{I S C O}$ of an irrotational sequence does not differ drastically from the frequency $f_{I S C O}^{s e c}$ determined from corotating calculations. Our results allow us to quantify this difference. For $n=0.5$ we find that the irrotational $f_{I S C O}$ is approximately $17 \%$ larger than the corresponding $f_{I S C O}^{s e c}$, while for $n=1$ the difference is approximately $20 \%$ (compare entries in Tables VII and VIII). For any polytropic index $n$, this difference depends only very weakly on $G M / R c^{2}$.

If multiple binary neutron star coalescence events are observed by instruments such as LIGO, then tables like Table VII, or figures like Figure 4 , will give information regarding the equation of state. For instance, consider a simple world in which binary components always had identical masses, although allow for varying neutron star mass from one binary to the next. Suppose further that all neutron stars obeyed the same (but unknown) polytropic equation of state. A single coalescence observation would yield a value of $M_{1.4} f_{I S C O}$, since the gravitational mass $M$ is encoded in the early inspiral waveform and since the ISCO frequency $f_{I S C O}$ can be determined by the onset of the orbital plunge. However, the equation of state and compactness parameter $G M / R c^{2}$ could not be uniquely determined from a single observation, since we do not yet know which curve (if either) in Figure 1 applies. For each value of $n$ being considered, the corresponding maximum gravitational mass $M^{\max }$ can be immediately determined from the observed value of $f_{I S C O}$ and from the $M_{1.6}^{\max } f_{I S C O}$ column in Table VII. An additional observation of a coalescence event with a different $f_{I S C O}$ would yield another value of $M^{\max }$ for each $n$. The polytropic index which consistently gives the same $M^{\max }$ regardless of the observed $f_{I S C O}$ must then represent the true equation of state. 
Tables similar to Table VII will therefore be helpful in testing realistic equations of state once coalescence observations are available.

There is only one equation of state for neutron stars, even if knowledge of it is still uncertain. For a given equation of state, one might ask how $f_{I S C O}$ depends on the mass of the binary components. For polytropes, fixing $n$ and $K$ also fixes the maximum gravitational mass $M^{\max }$ in isolation. From the $M_{1.6}^{\max } f_{I S C O}$ column of Table VII, we see that for $n=$ 0.5 the general trend of $f_{I S C O}$ is for it to decrease as the stellar mass $M$ (or $G M / R c^{2}$ ) increases, while for $n=1$ the frequency $f_{I S C O}$ increases with $M$. We therefore conclude that the relation between $f_{I S C O}$ and $M$ depends strongly on the equation of state. The reason for this dependence can be traced to two competing effects. Namely, as we consider more compact (larger $M$ ) stars, PN orbital destabilization effects become stronger, while Newtonian destabilization effects become weaker.

For the cases listed in Table VII, the total angular momentum $J$ is 0.96 to 1.10 times the total energy squared $M_{t}^{2}=\left(2 M_{o}+E\right)^{2}$ (in gravitational units, $\left.G=c=1\right)$ at the ISCO. In those cases with $J>M_{t}^{2}$, angular momentum would need to be either radiated away or carried away by ejected matter before a black hole can form (see Ref. [28] for a discussion). We find $J>M_{t}^{2}$ for all $n=0.5$ sequences with $G M / R c^{2} \lesssim 0.21$, while for $n=1$ this criterion is satisfied when $G M / R c^{2} \lesssim 0.18$.

Figure 7 presents the equilibrium rest mass $M_{o}$ as function of the central density for various orbital frequencies $f$ for an $n=1$ corotating sequence. We see that, for the terms we have included in our energy functional, the maximum mass in a close binary is slightly larger than in isolation. Figure 8 summarizes our maximum mass results for both irrotational and corotating sequences, and for $n=0.5$ and 1.0. The irrotational sequences maintain a lower maximum mass than their corresponding corotating sequences, because corotating stars (a) have more rotational support and (b) are more ellipsoidal so that the Newtonian tidal field has more of a stabilizing effect. The relative increases in $M_{o}^{\max }$ is more gradual for $n=0.5$ than $n=1.0$, because $n=0.5$ stars near the maximum mass are much more centrally condensed than $n=1.0$ stars near their maximum mass: from Table II we see 
that both the central density parameter $K \rho_{o c} / c^{2}$ and the compactness parameter $G M / R c^{2}$ are much larger for the maximum mass $n=0.5$ star $\left(\log q_{c}=0.12, G M / R c^{2}=0.39\right)$ than the $n=1$ star $\left(\log q_{c}=-0.39, G M / R c^{2}=0.26\right)$. Consequently, the $n=0.5$ stars near the maximum mass have smaller moments of inertia and behave more like point masses, so that stabilizing hydrodynamic effects play a less significant role. Our results suggest that the maximum equilibrium mass increases as the orbit decays, regardless of the sequence considered. However, if the maximum equilibrium mass does decrease in a binary system, it must be a result of the PN tidal terms, or of higher order PN corrections, that we are neglecting (see $\S \mathrm{VIQ})$.

\section{B. Comparison with Other Work}

Recently, Taniguchi and Nakamura 20] have applied the ellipsoidal approximation to a neutron star-black hole binary. They also compute irrotational and corotating sequences, but only for $n=0$ polytropes. In place of the hybrid-PN orbital energy contribution $E_{H}$, they use a generalized pseudo-Newtonian potential constructed to fit the ISCOs of the hybrid $\mathrm{P}^{2} \mathrm{~N}$ treatment of Kidder, Will, and Wiseman 4 . We improve upon the work of Taniguchi and Nakamura by including the relativistic corrections $U_{P N}$ and $W_{P N}$ to the internal energy of the neutron star, and by calculating sequences of fixed $M_{o} / R_{o}$, instead of fixed $M / R$ which varies as two neutron stars inspiral. Despite these differences our results do agree qualitatively: for a given equation of state more compact stars have larger ISCO frequencies, and for a fixed compactness parameter the ISCO along irrotational sequences is larger than the secular ISCO along corotating sequences.

Taniguchi and Shibata 22] have recently presented PN EFE analyses of binary neutron star systems which is rather similar to our own. The main differences are that (1) they derive equilibrium conditions from a tensor virial method, whereas we use an energy variational method, (2) they include all terms, up to quadrupole order, which are formally PN order, while we have neglected PN tidal terms and PN corrections to the internal fluid motion, (3) 
they consider only corotating sequences, while we also treat the more realistic irrotational sequences, and (4) they implement a simple Lane-Emden trial density function, as opposed to our PN-accurate density function [see eq. B19]. Our treatment does somewhat better than Taniguchi and Shibata's at matching the numerical, PN-accurate results of Shibata [29]. For instance, for $n=0.5$ and $G M_{o} / R_{o} c^{2}=0.02$, Shibata finds secular ISCO values of $\bar{E}, \bar{J}$, and $\bar{\Omega}$ equal to $-1.236,1.457$, and 0.31 , respectively; compare this to our values $-1.246,1.458$, and 0.316, and to Shibata and Taniguchi's values $-1.269,1.443$, and 0.327. We find a similar level of agreement with Shibata along other sequences: for $n=1.0$ and $G M_{o} / R_{o} c^{2}=1 / 60$, Shibata finds secular ISCO values of $\bar{E}, \bar{J}$, and $\bar{\Omega}$ equal to $-1.146,1.386$, and 0.35 , while our treatment gives $-1.155,1.384$, and 0.364 .

In order to examine the ISCO in irrotational sequences, Lai and Wiseman [21] have combined the Newtonian ellipsoidal equations of motion 13 with the hybrid $\mathrm{P}^{2} \mathrm{~N}$ point mass equations of motion of Kidder, Will, and Wiseman. This treatment does not include the PN coupling between the orbital motion and internal structure $\left(E_{O I}\right.$ and $J_{O I}$ in this paper), nor does it include PN corrections to the internal and self-gravitational energy $\left(U_{P N}\right.$ and $W_{P N}$ in this paper). Nevertheless, our results are in excellent agreement with those of Lai and Wiseman (see the comparison in Table $\nabla 1]$ ): for the three values of $G M / R_{o} c^{2}$ for which a direct comparison is possible, we find agreement to better than $4 \%$ in $f_{I S C O}$ and to better than about $2 \%$ in the ratios $a_{2} / a_{1}$ and $a_{3} / a_{1}$. The comparisons of $a_{2} / a_{1}$ and of $a_{3} / a_{1}$ should be viewed with some caution because of the freedom to measure distances with various coordinate systems in general relativity. In the Newtonian limit our results agree precisely with the Newtonian results of Lai and Wiseman [see their equations (8) and (9)], as they must since both treatments are extensions of the same Newtonian ellipsoidal approximation.

In the point mass limit, the results of Lai and Wiseman are accurate to $\mathrm{P}^{2} \mathrm{~N}$ order, whereas our results are accurate only to first PN order. We have not implemented the point mass $\mathrm{P}^{2} \mathrm{~N}$ orbital energy and angular momentum corrections in our energy functional, because doing so causes $d E=\Omega d J$ to hold to $\mathrm{P}^{2} \mathrm{~N}$ order but not exactly along our sequences, 
and because our functional would be neglecting other $\mathrm{P}^{2} \mathrm{~N}$ terms which are larger. However, we have explicitly tested the importance of the point mass $\mathrm{P}^{2} \mathrm{~N}$ corrections by adding them to our energy and angular momentum functions. We find that for $G M / R c^{2}=0.25$, the ISCO frequency for our $n=0.5$ and $n=1$ irrotational sequences is decreased by less than $4 \%$, while for $G M / R c^{2}=0.125$ and 0.2 this frequency is increased by about $2 \%$ or less.

Baumgarte et al. 18 have recently calculated in full general relativity quasi-equilibrium corotating sequences of binary neutron stars obeying a polytropic equation of state. Their computations are the most reliable to date for the neutron star structure, maximum equilibrium mass, and determination of the secular ISCO. Over the range of orbital separations they consider, they find the maximum equilibrium mass to increase slightly as the orbit decays, in agreement with the results presented in Figures 7 and 8. This agreement is reassuring, since these relativistic calculations essentially represent the "true" solution. Figure 9 compares our binding energy results with those of Ref. [18] for an $n=1$ corotating sequence.

The maximum equilibrium rest mass results presented in Figures 0 and 8 are also in qualitative agreement with the results of Lai [17]: the maximum mass of a neutron star seems to increase as the orbit decays. The main differences between our energy functional and that of Lai are that (a) Lai's adopted equation of state is based on a degenerate, nonrelativistic Fermi gas of nucleons ( $n=1.5$ polytrope), while we have concentrated on $n=0.5$ and $n=1$ polytropes, (b) Lai has included a correction $\Delta E_{\text {int }}$ as in Ref. 30 accounting for the tendency of neutrons to become mildly relativistic, and (c) we use equation (B44) for the self energy of a polytrope, whereas Lai uses equation (B47). Our maximum mass results are also completely consistent with the recent analytic treatments of Brady and Hughes [31] and of Wiseman [32], which show that there can be no decrease in the maximum equilibrium rest mass at first PN order, presuming tidal effects can be neglected. However, we cannot be sure that PN tidal effects would not change our results (see $\S \sqrt{\mathrm{VIO}}$ ). Nevertheless, our results are consistent with the relativistic integrations of Baumgarte et al. (1997), which automatically include all PN tidal terms. This agreement provides strong support of our analysis, at least in the case of corotating binaries. 


\section{Directions for Future Work}

Recently, Wilson, Mathews, and Marronetti [16] have presented numerical simulations which suggest that otherwise stable neutron stars may individually collapse to black holes when brought into a close binary orbit. Our results suggest that the maximum equilibrium mass increases as the orbit decays. In this section, however, we discuss two important caveats which leave open the possibility that the stars could collapse prior to the orbital plunge: (1) the PN tidal terms neglected in this paper may affect the maximum equilibrium mass, and (2) even if the maximum equilibrium mass does increase in binaries, some of the allowable equilibria may be unstable to collapse.

The largest uncertainty in our results may come from neglected PN tidal terms. Chandrasekhar [33] and Chandrasekhar and Nutku [34 have derived the PN corrections to the energy and angular momentum for arbitrary fluid configurations, which could be used to determine the PN corrections for our binary star system. The tidal components of these corrections are absent from our present analysis. For example, consider the PN correction from Ref. [34]'s equation (68) which is proportional to $\int \rho \Phi^{2} d^{3} x$, where $\Phi$ is the usual Newtonian gravitational potential. Inside a star, the potential $\Phi$ can be decomposed into an internal contribution $\Phi_{\text {int }}$ due to that star, and an external contribution $\Phi_{\text {ext }}$ due to the orbital companion. The integral $\int \rho \Phi_{\text {int }}^{2} d^{3} x$ then contributes to the self-gravitational PN energy $W_{P N}$, while the cross term $\int \rho \Phi_{\text {ext }} \Phi_{\text {int }} d^{3} x$ gives a coupling as in $E_{O I}$. The integral $\int \rho \Phi_{\text {ext }}^{2} d^{3} x$ to lowest order helps give the point mass PN orbital energy like in Ref. [4], and for finite-size stars this integral can be evaluated in a multipole expansion with the higher order terms accounting for PN tidal effects. A careful investigation of PN tidal effects seems worthwhile.

We also note that we have not tested our equilibrium configurations for stability against collapse to black holes: even if the maximum equilibrium mass does increase as the orbit decays, this does not necessarily mean the maximum stable mass follows the same trend. The EFE energy variational approach provides a straightforward means of testing the stability 
of equilibrium models, which could be exploited in future studies. In particular, the second order variation of the energy functional can be used to identify any instability to collapse.

\section{ACKNOWLEDGMENTS}

We would like to thank Phil Arras for a careful checking of the Appendices, as well as

Thomas Baumgarte, Éanna Flanagan, Larry Kidder, Dong Lai, Saul Teukolsky, and Alan Wiseman for useful discussions. This work was supported by NSF grant AST 91-19475 and NASA grant NAG5-2809 at Cornell, and by NSF grant AST 96-18524 and NASA grant NAG5-3420 at Illinois. FAR is supported by an Alfred P. Sloan Foundation Fellowship.

\section{APPENDIX A: POST-NEWTONIAN EXPANSION OF THE OV EQUATION}

In this appendix we expand the OV equation (see, eg. [30]) to PN order and thereby derive the first PN corrections to the Newtonian Lane-Emden functions for an isolated spherical polytrope in equilibrium. Our results are useful for generating trial density functions in $\S \mathrm{B} 2$ accurate to $\mathrm{PN}$ order.

Let $\rho_{o}(r)$ be the rest mass density of a spherically symmetric distribution of matter at the Schwarzschild radius $r$. Let $m(r)$ be the enclosed total mass-energy, and define the quantity $\xi$ and the non-dimensional functions $\vartheta(\xi)$ and $\nu(\xi)$ by

$$
\rho_{o}=\rho_{o c} \vartheta^{n}, \quad r=a \xi, \quad m=-4 \pi a^{3} \rho_{o c} \nu \xi^{2}
$$

where $\rho_{o c}=\rho_{o}(0)$ is the central rest mass density, so that $\vartheta(0)=1$. In the Newtonian limit, the functions $\vartheta(\xi)$ and $\nu(\xi)$ reduce to the usual Lane-Emden functions $\theta$ and $\theta^{\prime}$. Here $a=R / \xi_{I}$ is a scale factor, with $R$ being the radius of the star in Schwarzschild coordinates and $\xi_{I}$ being defined by the condition $\vartheta\left(\xi_{I}\right)=0$. For a polytropic equation of state

$$
P=K \rho_{o}^{1+\frac{1}{n}}
$$

the OV equations yield a hydrostatic equilibrium solution if (see, eg., Ref. [35]) 


$$
\begin{aligned}
\frac{d \vartheta}{d \xi} & =\frac{\nu\left(1+(n+1) q_{c} \vartheta\right)\left(1-q_{c} \vartheta^{n+1} \xi / \nu\right)}{\left(1+2(n+1) q_{c} \nu \xi\right)}, \\
\frac{1}{\xi^{2}} \frac{d\left(\nu \xi^{2}\right)}{d \xi} & =-\vartheta^{n}\left(1+n q_{c} \vartheta\right), \\
\frac{K \rho_{o c}^{1 / n}}{c^{2}} & =q_{c}
\end{aligned}
$$

where we have defined

$$
q_{c} \equiv \frac{4 \pi \rho_{o c} G a^{2}}{(n+1) c^{2}} .
$$

Evaluating the last equation of (A1) at the surface, we see that for an equilibrium configuration the scale factor $a$ satisfies

$$
4 \pi a^{3} \rho_{o c}=\frac{M}{\xi_{I}^{2}\left|\nu_{I}\right|},
$$

where $M$ is the total mass-energy and $\nu_{I}=\nu\left(\xi_{I}\right)$. In the Newtonian limit $(c \rightarrow \infty), q_{c} \rightarrow 0$, $\vartheta \rightarrow \theta$ and $\nu \rightarrow \theta^{\prime}$, where $\theta$ is the usual Lane-Emden function. If we define the functions $\alpha$ and $\beta$ as the $\mathrm{PN}$ components in the expansions

$$
\begin{aligned}
& \vartheta=\theta+q_{c} \alpha+\mathcal{O}\left(q_{c}^{2}\right), \\
& \nu=\theta^{\prime}+q_{c} \beta+\mathcal{O}\left(q_{c}^{2}\right),
\end{aligned}
$$

then equations ( $\mathrm{A3})$ and (A4) become

$$
\begin{aligned}
\frac{d \alpha}{d \xi} & =\beta+(n+1) \theta \theta^{\prime}-\theta^{n+1} \xi-2(n+1)\left(\theta^{\prime}\right)^{2} \xi, \\
\frac{1}{\xi^{2}} \frac{d\left(\beta \xi^{2}\right)}{d \xi} & =-n\left(\alpha \theta^{n-1}+\theta^{n+1}\right) .
\end{aligned}
$$

In deriving equations (A10) and (A11) we have used the Lane-Emden equation to cancel all terms of Newtonian order. We note that the functions $\alpha$ and $\beta$ depend only on the polytropic index $n$ and can be numerically integrated simultaneously with the Lane-Emden equation. The numerical integrations can be started slightly away from $\xi=0$ with the help of the approximations, for $\xi<<1$ :

$$
\begin{aligned}
& \alpha \approx-\frac{\xi^{2}}{3}(n+2), \\
& \beta \approx-\frac{n}{3} \xi .
\end{aligned}
$$


The surface occurs when $0=\vartheta\left(\xi_{I}\right)=\theta\left(\xi_{I}\right)+q_{c} \alpha\left(\xi_{I}\right)=\left(\xi_{I}-\xi_{1}\right) \theta_{1}^{\prime}+q_{c} \alpha\left(\xi_{1}\right)$, to PN order, where we have Taylor expanded and used $\theta\left(\xi_{1}\right)=0$ to obtain the last equality. Therefore,

$$
\xi_{I}=\xi_{1}-q_{c} \frac{\alpha_{1}}{\theta_{1}^{\prime}}+\mathcal{O}\left(q_{c}^{2}\right)
$$

where $\alpha_{1}=\alpha\left(\xi_{1}\right)$. Similarly, Taylor expanding equation (A9) about the surface at $\xi=\xi_{I}$ gives

$$
\nu_{I}=\theta_{1}^{\prime}+q_{c} \frac{2 \alpha_{1}}{\xi_{1}}+q_{c} \beta_{1}+\mathcal{O}\left(q_{c}^{2}\right)
$$

where $\nu_{I}=\nu\left(\xi_{I}\right)$ and $\beta_{1}=\beta\left(\xi_{1}\right)$.

In the special case $n=1$ it is well known that the solution to the Lane-Emden equation is $\theta=\sin (\xi) / \xi$. Equations (A10) and (A11) can also be solved analytically when $n=1$ :

$$
\begin{aligned}
& \alpha=\theta^{2}-\theta-2 \xi \theta^{\prime} \theta-3 \xi^{2} \theta^{4}-3 \theta \int_{0}^{\xi} \xi \theta^{3} d \xi+9\left(\theta^{\prime}+\frac{\theta}{\xi}\right) \int_{0}^{\xi} \xi^{2} \theta^{3} d \xi \\
& \beta=3 \xi \theta^{2}-\theta^{\prime}+2 \theta \theta^{\prime}-3 \xi^{2} \theta^{3} \theta^{\prime}+2 \xi \theta^{\prime 2}-3 \theta^{\prime} \int_{0}^{\xi} \xi \theta^{3} d \xi-\frac{9\left(\theta+\xi^{2} \theta+\xi \theta^{\prime}\right)}{\xi^{2}} \int_{0}^{\xi} \xi^{2} \theta^{3} d \xi
\end{aligned}
$$

These equations provide a convenient check of our numerical integrations in Appendix B.

\section{APPENDIX B: POST-NEWTONIAN SELF-ENERGY TERMS}

We now derive the PN self energy terms of a spherically symmetric fluid which is instantaneously at rest. Our approach generalizes the method of Ref. [36]. Let us introduce the enclosed rest mass $m^{\prime}$ and the proper radial coordinate $r^{\prime}$, which are related to the enclosed total mass-energy $m$ and the Schwarzschild radius $r$ by

$$
d m^{\prime}=\rho_{o} 4 \pi r^{\prime 2} d r^{\prime}=\left(1-\frac{2 G m}{r c^{2}}\right)^{-1 / 2} \rho_{o} 4 \pi r^{2} d r=\left(1-\frac{2 G m}{r c^{2}}\right)^{-1 / 2}\left(1+\frac{u}{c^{2}}\right)^{-1} d m
$$

where $u$ is the internal energy density. To PN order, the energy of such a system, excluding rest mass energy, is [see equations (6.9.3), (6.9.8), (6.9.10) and (6.9.18) of Ref. [30]]

$$
\begin{aligned}
E & =c^{2} \int_{0}^{M} d m-c^{2} \int_{0}^{M_{o}} d m^{\prime} \\
& =\int_{0}^{M_{o}} u d m^{\prime}-G \int_{0}^{M_{o}} \frac{m^{\prime}}{r^{\prime}} d m^{\prime}+I_{1}+I_{2}+I_{3}+I_{4}+I_{5},
\end{aligned}
$$


where $M_{o}$ is the total rest mass, and

$$
\begin{aligned}
& I_{1}=-\frac{G}{c^{2}} \int_{0}^{M} u \frac{m}{r} d m, \\
& I_{2}=-\frac{G^{2}}{2 c^{2}} \int_{0}^{M}\left(\frac{m}{r}\right)^{2} d m, \\
& I_{3}=-\frac{G}{c^{2}} \int_{0}^{M} \frac{d m}{r} \int_{0}^{m} u d m, \\
& I_{4}=\frac{G^{2}}{c^{2}} \int_{0}^{M} \frac{d m}{r} \int_{0}^{m} \frac{m}{r} d m, \\
& I_{5}=-\frac{G^{2}}{c^{2}} \int_{0}^{M} \frac{m d m}{r^{4}} \int_{0}^{r} m r d r .
\end{aligned}
$$

Since we are working only to PN order, the quantities $m$ and $r$ can be replaced with $m^{\prime}$ and $r^{\prime}$ in $I_{1}$ to $I_{5}$, if desired.

Note that equations ( $\mathrm{B} 2)$ and $(\overline{\mathrm{B} 3})$ do not require that the fluid be in equilibrium: the density profile $\rho_{o}$ and internal energy profile $u$ need to be spherically symmetric, but are otherwise arbitrary. Let us now restrict our attention to fluids obeying a polytropic equation of state

$$
P=K \rho_{o}^{1+\frac{1}{n}}, \quad u=n K \rho_{o}^{1 / n}
$$

where $K$ is a constant, but allow the density profile $\rho_{o}$ to remain arbitrary for the moment. We wish to find the contribution to the energy beyond that given by the usual Newtonian expressions for the internal energy $U$ and the self-gravitational energy $W$ [see equations (12) and (13)], ie.

$$
\Delta E \equiv E-\left(k_{1} K \rho_{o c}^{1 / n} M_{o}-k_{2} G \rho_{o c}^{1 / 3} M_{o}^{5 / 3}\right)
$$

where it is understood that all terms of order $\mathrm{P}^{2} \mathrm{~N}$ and higher are dropped. Inspection of equations $(\mathbb{B} 3),(\mathbb{B 9})$ and $(\overline{B 10})$ reveals that $\Delta E$ is composed of two types of terms: those which depend on $K$ and those which do not; we write

$$
\Delta E=U_{P N}+W_{P N},
$$

where we have defined 


$$
\begin{aligned}
U_{P N} & =\int_{0}^{M_{o}} u d m^{\prime}-k_{1} K \rho_{o c}^{1 / n} M_{o}+I_{1}+I_{3}, \\
W_{P N} & =-G \int_{0}^{M_{o}} \frac{m^{\prime}}{r^{\prime}} d m^{\prime}+k_{2} G \rho_{o c}^{1 / 3} M_{o}^{5 / 3}+I_{2}+I_{4}+I_{5} .
\end{aligned}
$$

The quantity $U+W+U_{P N}+W_{P N}$ is the energy of a spherical symmetric configuration with an arbitrary density profile, to PN order.

If we restrict our attention to a set of well-chosen density functions, then the integrals in equations $(\overline{B 12})$ and $(\overline{B 13})$ can be evaluated. In particular, if we consider a family of density profiles of the form $\rho_{o}\left(r^{\prime}\right) \propto f\left(r^{\prime}\right)$ [or equivalently $\rho_{o}(r) \propto g(r)$, where $f\left(r^{\prime}\right)$ [or $g(r)$ ] is arbitrary, then

$$
\begin{aligned}
U_{P N} & =-l_{1} \frac{G}{c^{2}} K \rho_{o c}^{\frac{1}{n}+\frac{1}{3}} M_{o}^{5 / 3}, \\
W_{P N} & =-l_{2} \frac{G^{2}}{c^{2}} \rho_{o c}^{2 / 3} M_{o}^{7 / 3} .
\end{aligned}
$$

Here the dimensionless coefficients $l_{1}$ and $l_{2}$ are determined by evaluating the integrals in equations (B12) and (B13) for the chosen family of density profiles.

\section{Lane-Emden Trial Density Functions}

As a concrete example, consider density profiles which are related to the non-dimensional Lane-Emden function $\theta$ by

$$
\rho_{o}^{\mathrm{LE}}\left(r^{\prime}\right)=\rho_{o c} \theta^{n}, \quad r^{\prime}=\frac{R^{\prime}}{\xi_{1}} \xi
$$

where $R^{\prime}$ is the proper radius of the star and the central density $\rho_{o c}$ does not necessarily have the value which would allow for equilibrium at Newtonian order. The superscript "LE" appearing in equation $(\mathbb{B 1 6})$ is a reminder that we are limiting ourselves to a single family of trial density profiles based upon Lane-Emden functions. Since density profiles of this form differ at least at PN order from the OV solution which minimizes the energy given by 
equation (B2), there is no value of $\rho_{o c}$ for which the fluid is in equilibrium to PN order.t Using equation $(\mathrm{B16})$ in equations $(\mathrm{B12})$ and $(\mathrm{B13})$ we obtain simply

$$
\begin{aligned}
l_{1}^{\mathrm{LE}} & =\frac{I_{1}+I_{3}}{-\frac{G}{c^{2}} K \rho_{o c}^{\frac{1}{n}+\frac{1}{3}} M_{o}^{5 / 3}}, \\
l_{2}^{\mathrm{LE}} & =\frac{I_{2}+I_{4}+I_{5}}{-\frac{G^{2}}{c^{2}} \rho_{o c}^{2 / 3} M_{o}^{7 / 3}} .
\end{aligned}
$$

From here it is straightforward to write $l_{1}^{\mathrm{LE}}$ and $l_{2}^{\mathrm{LE}}$ as integrals over Lane-Emden functions, and these coefficients therefore depend only on the polytropic index $n$. Equations (B17) and (B18) define the coefficients used to produce the short dashed curves in Figure 1.

In the following subsection we go through a considerable amount of work to derive $l_{1}$ and $l_{2}$ for more accurate trial density functions. However, the coefficients given by equations (B17) and (B18) already represent a significant improvement over that used previously in the literature (compare the short-dashed and dot-dashed curves in Figure 1). The reason for this improvement is that we have kept both of the PN terms [eqs. (B14) and (B15)] which result naturally from the subtraction in equation (B10). Previous analyses have used equilibrium relations to eliminate $K$ in equation (B14) and forced $U_{P N}$ to scale like $W_{P N}$, resulting in an energy functional which is valid only along equilibrium sequences and therefore should not be used in an energy variational treatment [see the discussion surrounding eq. (B47) below]. Nowhere in the derivation of equations $(\overline{B 17})$ and $(\overline{B 18})$ have we assumed the star is in equilibrium.

\section{OV-Based Trial Density Functions}

The disadvantage of being restricted to Lane-Emden trial density functions is that the energy minimum of equation (B3) then differs at PN order from the actual energy minimum. We know that fluid configurations which obey the OV equation are precisely those necessary

\footnotetext{
${ }^{1}$ The exception to this statement occurs for $n=0$. In this case, both the purely Newtonian and the exact general relativistic density profile are of the form $\rho_{o}=$ const.
} 
to minimize the energy given by equation (B2). This suggests that we construct a family of trial density functions based upon the solution to the OV equation, namely

$$
\rho_{o}(r)=\rho_{o c}\left(\theta+q_{c} \alpha\right)^{n}, \quad r=\frac{R}{\xi_{I}} \xi
$$

where $R$ is the stellar radius in Schwarzschild coordinates, the central density $\rho_{o c}$ is not necessarily the value required by the OV equation for equilibrium [ie. eqs. (A5) and (A7) need not be satisfied], and the function $\alpha$ is obtained by solving equations (A10) and (A11). The expansion parameter $q_{c}$ is defined by equation (A6) with $a=R / \xi_{I}$, ie.

$$
q_{c}=\frac{4 \pi \rho_{o c} G R^{2}}{\xi_{I}^{2}(n+1) c^{2}} .
$$

An energy variational method based upon this family of trial density functions can identify the minimum of equation (B3) to PN order. There are an infinite number of other families which satisfy this criterion [for instance, we could generate one other such family by replacing $\rho_{o c}$ with its equilibrium value in eq. (B20)]. However, the trial density functions defined by equations $(\mathrm{B} 19)$ and $(\mathrm{B} 20)$ are independent of the polytropic constant $K$ and hence make it easy to track which terms do and do not depend on $K$. We note that the last equation of (A1) does not apply here, although by construction it is satisfied to PN order when $\rho_{o c}$ has its equilibrium value. [To derive the enclosed total mass-energy even for non-equilibrium configurations, use $m=\int \rho 4 \pi r^{2} d r$ with $\rho=\rho_{o}\left(1+u / c^{2}\right)$.]

Our plan is now to evaluate $l_{1}$ and $l_{2}$ as defined by equations (B14) and (B15), using the family of trial density functions given in equation (B19). We begin with the first integral appearing in equation (B12) and work to $\mathrm{PN}$ order:

$$
\begin{aligned}
\int_{0}^{M_{o}} u d m^{\prime} & =\int_{0}^{M} u\left(1-\frac{2 G m}{r c^{2}}\right)^{-1 / 2}\left(1+\frac{u}{c^{2}}\right)^{-1} d m \\
& =\int_{0}^{M} u\left(1+\frac{G m}{r c^{2}}-\frac{u}{c^{2}}\right) d m \\
& =\int_{0}^{M} u\left(1-\frac{u}{c^{2}}\right) d m-I_{1},
\end{aligned}
$$

where we have used equation ( 


$$
\begin{aligned}
\int_{0}^{M} u\left(1-\frac{u}{c^{2}}\right) d m & =4 \pi n \int_{0}^{R} \operatorname{Pr}^{2} d r \\
& =n K \rho_{o c}^{1 / n} \frac{4 \pi R^{3} \rho_{o c}}{\xi_{I}^{3}} \int_{0}^{\xi_{I}}\left(\theta+q_{c} \alpha\right)^{n+1} \xi^{2} d \xi \\
& =n K \rho_{o c}^{1 / n} \frac{4 \pi R^{3} \rho_{o c}}{\xi_{I}^{3}} \int_{0}^{\xi_{I}} \theta^{n+1}\left(1+(n+1) \frac{q_{c} \alpha}{\theta}\right) \xi^{2} d \xi \\
& =n K \rho_{o c}^{1 / n} \frac{4 \pi R^{3} \rho_{o c}}{\xi_{I}^{3}}\left(\int_{0}^{\xi_{I}} \theta^{n+1} \xi^{2} d \xi+(n+1) q_{c} \int_{0}^{\xi_{I}} \alpha \theta^{n} \xi^{2} d \xi\right) .
\end{aligned}
$$

Note that since we are working only to PN order, it is justified to end all the integrals in this appendix at $\xi_{1}$ [where $\xi_{1}$ is defined by $\left.\theta\left(\xi_{1}\right)=0\right]$. For instance, since $\theta \lesssim q_{c}$ in the range $\xi_{I}<\xi<\xi_{1}$, and since $\xi_{1}-\xi_{I} \sim q_{c}$,

$$
\int_{0}^{\xi_{I}} \theta^{n+1} \xi^{2} d \xi=\int_{0}^{\xi_{1}} \theta^{n+1} \xi^{2} d \xi+\mathcal{O}\left(q_{c}^{n+2}\right)=\frac{k_{1}}{n} \xi_{1}^{2}\left|\theta_{1}^{\prime}\right|+\mathcal{O}\left(q_{c}^{n+2}\right) .
$$

Therefore equation (B27) becomes

$$
\int_{0}^{M} u d m=k_{1} K \rho_{o c}^{1 / n} \xi_{1}^{2}\left|\theta_{1}^{\prime}\right| \frac{4 \pi R^{3} \rho_{o c}}{\xi_{I}^{3}}\left(1+\frac{n(n+1) q_{c}}{k_{1} \xi_{1}^{2}\left|\theta_{1}^{\prime}\right|} \int_{0}^{\xi_{1}} \alpha \theta^{n} \xi^{2} d \xi\right)
$$

In order to write our final answer in the form of equation (B11), we need to derive a relation between $\left(4 \pi R^{3} \rho_{o c} / \xi_{I}^{3}\right)$ and the total rest mass $M_{o}$. From our knowledge of Newtonian polytropes we expect $\left(4 \pi R^{3} \rho_{o c} / \xi_{I}^{3}\right)=M_{o} /\left(\xi_{1}^{2}\left|\theta_{1}^{\prime}\right|\right)+\mathcal{O}\left(q_{c}\right)$, and we can obtain a relation accurate to PN order by evaluating $M_{o}$ :

$$
\begin{aligned}
M_{o} & =\int_{0}^{M_{o}} d m^{\prime} \\
& =\int_{0}^{R} \rho_{o}\left(1-\frac{2 G m}{r c^{2}}\right)^{-1 / 2} 4 \pi r^{2} d r \\
& =\frac{4 \pi R^{3} \rho_{o c}}{\xi_{I}^{3}} \int_{0}^{\xi_{I}}\left(\theta+q_{c} \alpha\right)^{n}\left(1+\frac{G m}{r c^{2}}\right) \xi^{2} d \xi \\
& =\frac{4 \pi R^{3} \rho_{o c}}{\xi_{I}^{3}} \int_{0}^{\xi_{I}} \theta^{n}\left(1+n \frac{q_{c} \alpha}{\theta}+\frac{4 \pi G \rho_{o c} R^{2} \xi\left|\theta^{\prime}\right|}{\xi_{I}^{2} c^{2}}\right) \xi^{2} d \xi \\
& =\frac{4 \pi R^{3} \rho_{o c}}{\xi_{I}^{3}}\left(\int_{0}^{\xi_{I}} \theta^{n} \xi^{2} d \xi+q_{c} \int_{o}^{\xi_{I}}\left(n \alpha \theta^{n-1} \xi^{2}-(n+1) \theta^{n} \theta^{\prime} \xi^{3}\right) d \xi\right) .
\end{aligned}
$$

Making use of

$$
\int_{0}^{\xi_{I}} \theta^{n} \xi^{2} d \xi=\xi_{1}^{2}\left|\theta_{1}^{\prime}\right|+\mathcal{O}\left(q_{c}^{n+1}\right), \quad \int_{0}^{\xi_{I}} \theta^{n} \theta^{\prime} \xi^{3} d \xi=\frac{-3}{5-n} \xi_{1}^{3}\left|\theta_{1}^{\prime}\right|^{2}+\mathcal{O}\left(q_{c}^{n+1}\right)
$$


in equation (B34) gives

$$
\begin{aligned}
\frac{4 \pi R^{3} \rho_{o c}}{\xi_{I}^{3}} & =\frac{M_{o}}{\xi_{1}^{2}\left|\theta_{1}^{\prime}\right|}\left(1-\frac{n q_{c}}{\xi_{1}^{2}\left|\theta_{1}^{\prime}\right|} \int_{0}^{\xi_{I}} \alpha \theta^{n-1} \xi^{2} d \xi-\frac{3(n+1)}{5-n} q_{c} \xi_{1}\left|\theta_{1}^{\prime}\right|\right) \\
& =\frac{M_{o}}{\xi_{1}^{2}\left|\theta_{1}^{\prime}\right|}\left(1+q_{c} \frac{\beta_{1}}{\left|\theta_{1}^{\prime}\right|}+q_{c} \frac{(n-3)(n+1)}{5-n} \xi_{1}\left|\theta_{1}^{\prime}\right|\right)
\end{aligned}
$$

where $\beta_{1}=\beta\left(\xi_{1}\right)$. We evaluated the integral in equation (B36) with the help of equations ( $\mathrm{A11})$ and $(\overline{\mathrm{B} 28})$. Equation ( $\mathrm{B} 37)$ is our desired relation between $\left(4 \pi R^{3} \rho_{o c} / \xi_{I}^{3}\right)$ and $M_{o}$.

Using equations $(\mathbb{B} 37)$ and $(\bar{B} 29)$ in equation $(\bar{B} 23)$ gives

$$
\begin{aligned}
\int_{0}^{M_{o}} u d m^{\prime}= & k_{1} K \rho_{o c}^{1 / n} M_{o}\left(1+\frac{n(n+1) q_{c}}{k_{1} \xi_{1}^{2}\left|\theta_{1}^{\prime}\right|} \int_{0}^{\xi_{1}} \alpha \theta^{n} \xi^{2} d \xi+q_{c} \frac{\beta_{1}}{\left|\theta_{1}^{\prime}\right|}\right. \\
& \left.+q_{c} \frac{(n-3)(n+1)}{5-n} \xi_{1}\left|\theta_{1}^{\prime}\right|\right)-I_{1} .
\end{aligned}
$$

We note that the lowest order term is the usual internal energy contribution for a pure polytrope, $k_{1} K \rho_{o c}^{1 / n} M_{o}$, as expected; the first order corrections will help give us our expression for the coefficient $l_{1}$. Using equation $(\mathrm{B} 38)$ and the definition of $k_{1} \equiv n(n+1) \xi_{1}\left|\theta_{1}^{\prime}\right| /(5-n)$ in equation ( $\mathrm{B} 12)$ gives

$$
\begin{aligned}
U_{P N}= & \frac{K \rho_{o c}^{1 / n} M_{o} n(n+1) q_{c}}{\xi_{1}^{2}\left|\theta_{1}^{\prime}\right|}\left(\int_{0}^{\xi_{1}} \alpha \theta^{n} \xi^{2} d \xi+\frac{\beta_{1} \xi_{1}^{3}\left|\theta_{1}^{\prime}\right|}{(5-n)}\right. \\
& \left.+\frac{(n-3)(n+1)}{(5-n)^{2}} \xi_{1}^{4}\left|\theta_{1}^{\prime}\right|^{3}\right)+I_{3},
\end{aligned}
$$

or, after using equations (B20) and (B37) to lowest order and simplifying,

$$
\begin{aligned}
l_{1}= & \frac{(4 \pi)^{1 / 3}}{\left(\xi_{1}^{2}\left|\theta_{1}^{\prime}\right|\right)^{5 / 3}} n\left(-\int_{0}^{\xi_{1}} \alpha \theta^{n} \xi^{2} d \xi-\frac{\beta_{1} \xi_{1}^{3}\left|\theta_{1}^{\prime}\right|}{(5-n)}\right. \\
& \left.-\frac{(n-3)(n+1)}{(5-n)^{2}} \xi_{1}^{4}\left|\theta_{1}^{\prime}\right|^{3}+\int_{0}^{\xi_{1}} d \xi \theta^{n} \xi \int_{0}^{\xi} \theta^{n+1} \xi^{2} d \xi\right) .
\end{aligned}
$$

The relation'2

\footnotetext{
${ }^{2}$ To prove this relation, begin by non-dimensionalizing the double integral $I_{3}$ and writing it in terms of the single integrals $I_{1}$ and $I_{2}$ in non-dimensional form, following the strategy of Exercise 6.19 in Ref. [30]. Then with repeated integration by parts and use of the Lane-Emden equation, the two remaining integrals can be written in terms of each other, and the identity is obtained.
} 


$$
\int_{0}^{\xi_{1}} d \xi \theta^{n} \xi \int_{0}^{\xi} \theta^{n+1} \xi^{2} d \xi=\frac{n+1}{5-n} \xi_{1}^{4}\left|\theta_{1}^{\prime}\right|^{3}-\frac{n-1}{3} \int_{0}^{\xi_{1}} \xi^{3} \theta^{\prime} \theta^{n+1} d \xi
$$

can be used to reduce the double integral in equation (B40) to a single integral:

$$
\begin{aligned}
& l_{1}=\frac{(4 \pi)^{1 / 3}}{\left(\xi_{1}^{2}\left|\theta_{1}^{\prime}\right|\right)^{5 / 3}} n\left(-\frac{n-1}{3} \int_{0}^{\xi_{1}} \xi^{3} \theta^{\prime} \theta^{n+1} d \xi\right. \\
& \left.-\int_{0}^{\xi_{1}} \alpha \theta^{n} \xi^{2} d \xi-\frac{\beta_{1} \xi_{1}^{3}\left|\theta_{1}^{\prime}\right|}{5-n}-2 \frac{(n-4)(n+1)}{(5-n)^{2}} \xi_{1}^{4}\left|\theta_{1}^{\prime}\right|^{3}\right) \text {. }
\end{aligned}
$$

If desired, the identity [which can be proved from equations (A10) and (A11) and appropriate integrations by parts]

$$
\int_{0}^{\xi_{1}} \alpha \theta^{n} \xi^{2} d \xi=\frac{1}{1-n}\left[\alpha_{1} \xi_{1}^{2}\left|\theta_{1}^{\prime}\right|-\frac{1}{6}\left(n^{2}+25 n+28\right) \int_{0}^{\xi_{1}} \xi^{3} \theta^{\prime} \theta^{n+1} d \xi\right]
$$

could be used to further manipulate equation ( $(\overline{\mathrm{B} 42})$ when $n \neq 1$, while for $n=1$ this integral can be evaluated using equation (A16). However, we find it more convenient to work with equation (B42) directly.

Equation (B42) is our final expression for the coefficient $l_{1}$, and was obtained by evaluating equation (B12) with the OV-based trial density functions defined in equation (B19). We could proceed to calculate $l_{2}$ from equation $(\mathbb{B} 13)$ in a similar manner. However, it is more straightforward to take the following approach.

Rearranging equations $(\mathbb{B 1 0})$ and $(\mathbb{B 1 1})$ yields the PN accurate energy functional for an isolated spherical star, not necessarily in equilibrium, obeying a polytropic equation of state:

$$
E=k_{1} K \rho_{o c}^{1 / n} M_{o}-k_{2} G \rho_{o c}^{1 / 3} M_{o}^{5 / 3}-l_{1} \frac{G}{c^{2}} K \rho_{o c}^{\frac{1}{n}+\frac{1}{3}} M_{o}^{5 / 3}-l_{2} \frac{G^{2}}{c^{2}} \rho_{o c}^{2 / 3} M_{o}^{7 / 3}
$$

Along an equilibrium sequence we have $0=d E / d \rho_{o c}$, which gives, to lowest order,

$$
K \rho_{o c}^{1 / n}=\frac{n k_{2}}{3 k_{1}} G M_{o}^{2 / 3} \rho_{o c}^{1 / 3}
$$

Indeed, equation $(\mathrm{B} 45)$ is the same relation obtained by writing the Newtonian equation of hydrostatic equilibrium in terms of Lane-Emden functions and simplifying. To PN order, the energy along an equilibrium sequence therefore satisfies

$$
E^{e q}=k_{1} K \rho_{o c}^{1 / n} M_{o}-k_{2} G \rho_{o c}^{1 / 3} M_{o}^{5 / 3}-\left(l_{1} \frac{n k_{2}}{3 k_{1}}+l_{2}\right) \frac{G^{2}}{c^{2}} \rho_{o c}^{2 / 3} M_{o}^{7 / 3} .
$$


Since here we are focusing on the equilibrium sequence, the first variational derivative of $E$ with respect to the density functional must vanish; therefore, any variation in the density profile of $\mathrm{PN}$ order causes a variation in the energy of order $\mathrm{P}^{2} \mathrm{~N}$, so that it is sufficient to use Lane-Emden profiles when evaluating the Newtonian order integrals in $\Delta E$. We therefore find that along an equilibrium sequence $\Delta E=I_{1}+I_{2}+I_{3}+I_{4}+I_{5}$. The Newtonian equation of hydrostatic equilibrium, equation $(\bar{B} 45)$, can then be used to evaluate $I_{1}$ through $I_{5}$ in this case. These integrals have been carried out by Ref. [30] (see their §6.9), so that

$$
E^{e q}=k_{1} K \rho_{o c}^{1 / n} M_{o}-k_{2} G \rho_{o c}^{1 / 3} M_{o}^{5 / 3}-k \frac{G^{2}}{c^{2}} \rho_{o c}^{2 / 3} M_{o}^{7 / 3},
$$

where (see eq. [6.9.31] of [30])

$$
\begin{aligned}
k & =\frac{(4 \pi)^{2 / 3}}{(5-n)\left[\xi_{1}^{2}\left|\theta_{1}^{\prime}\right|\right]^{7 / 3}}\left(-\frac{5+2 n-n^{2}}{n+1} 2 \int_{0}^{\xi_{1}} \xi^{3} \theta^{\prime} \theta^{n+1} d \xi+\frac{3}{2}(n-1) \int_{0}^{\xi_{1}} \xi^{4} \theta^{\prime 2} \theta^{n} d \xi\right) \\
& =\frac{(4 \pi)^{2 / 3}}{\left[\xi_{1}^{2}\left|\theta_{1}^{\prime}\right|\right]^{7 / 3}}\left(\frac{n-1}{2(5-n)} \xi_{1}^{4}\left|\theta_{1}^{\prime}\right|^{3}-\frac{(n+2)(n+5)}{6(n+1)} \int_{0}^{\xi_{1}} \xi^{3} \theta^{\prime} \theta^{n+1} d \xi\right) .
\end{aligned}
$$

By integrating the OV equation exactly and extracting coefficients from the resulting equilibrium sequence energy $E^{e q}\left(\rho_{o c}\right)$, we have numerically verified that equation (B47) holds to $\mathrm{PN}$ order. We emphasize that equation (B46) or (B47), in contrast to equation (B44), should not be used as energy functionals in an energy variational treatment, since they are valid only for equilibrium configurations ${ }^{3}$; this has not been noticed in previous analyses.

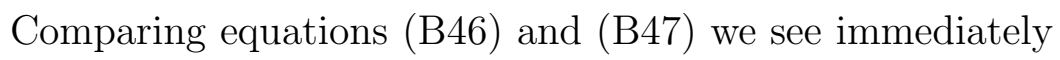

$$
l_{2}=k-l_{1} \frac{n k_{2}}{3 k_{1}}
$$

\footnotetext{
${ }^{3}$ In the special case $n=0$, the self energy correction $U_{P N}=0$, and the use of equation (B47) as an energy functional does yield correct answers to first PN order. Equation (B47) also yields PN accurate answers when $n=3$; this is because $U_{P N}$ and $W_{P N}$ both then scale as $\rho_{o c}^{2 / 3}$ and because the equilibrium relation between $K$ and $M_{o}$ is independent of $\rho_{o c}$ at lowest order [see eq. (B45)]. However, for $n \neq 0$ and $n \neq 3$, use of equation $(\overline{\mathrm{B} 47})$ as an energy functional gives errors at first PN order.
} 


$$
\begin{aligned}
= & k-\frac{l_{1}}{n+1}\left(\frac{4 \pi}{\xi_{1}^{4}\left|\theta_{1}^{\prime}\right|^{2}}\right)^{1 / 3} \\
= & \frac{(4 \pi)^{2 / 3}}{\left[\xi_{1}^{2}\left|\theta_{1}^{\prime}\right|\right]^{7 / 3}}\left(\frac{n-10}{6} \int_{0}^{\xi_{1}} \xi^{3} \theta^{\prime} \theta^{n+1} d \xi\right. \\
& \left.+\frac{n}{n+1} \int_{0}^{\xi_{1}} \alpha \theta^{n} \xi^{2} d \xi+\frac{n \beta_{1} \xi_{1}^{3}\left|\theta_{1}^{\prime}\right|}{(n+1)(5-n)}+\frac{3 n^{2}-10 n-5}{2(5-n)^{2}} \xi_{1}^{4}\left|\theta_{1}^{\prime}\right|^{3}\right) .
\end{aligned}
$$

Equations (B42) and (B52) are our final expressions for the coefficients $l_{1}$ and $l_{2}$, and were used to generate the long dashed curves in Figure 1. The Newtonian curves in this figure, as well as the $n=1$ curves which use equation (B47), have a maximum in the gravitational mass $M$ which is not accompanied by a maximum in the rest mass $M_{o}$; this is because along these sequences $d M / d \rho_{o c}=\left(\partial M / \partial M_{o}\right)\left(d M_{o} / d \rho_{o c}\right)$ vanishes due to $\partial M / \partial M_{o}=0$. The remaining sequences have maxima in $M$ and $M_{o}$ at the same $\rho_{o c}$, where $0=d M / d \rho_{o c}=d M_{o} / d \rho_{o c}$, and these turning points mark the onset of radial instability.

The derivation of the coefficients $l_{1}$ and $l_{2}$ is formally invalid if $n=0$, since then the first relation in equation (B35) is accurate only to Newtonian order. However, for $n=0$ one can analytically derive the coefficients exactly to be $l_{1}=0$ and $l_{2}=3(4 \pi / 3)^{2 / 3} / 70$, and the numerically obtained values given by equations (B42) and (B52) do indeed approach these limiting values as $n$ approaches zero. See Table [ for numerical values of these coefficients for various $n$. We note that the coefficient $l_{1}$ is fairly well approximated simply by $l_{1} \approx n$. Also note that for $n \gtrsim 0.5, l_{1}$ is roughly 10 or more times larger in magnitude than $l_{2}$, so that we expect $U_{P N}$ to be significantly larger than $W_{P N}$ in magnitude for all but the stiffest equations of state. 


\section{REFERENCES}

[1] D. J. Nice, R. W. Sayer, and J. H. Taylor, Astrophys. J. 466, L87 (1996).

[2] R. Narayan, B. Paczyński, and T. Piran, Astrophys. J. 395, L83 (1992).

[3] R. Epstein and R. V. Wagoner, Astrophys. J. 197, 717 (1975); C. W. Lincoln and C. M. Will, Phys. Rev. D. 42, 1123 (1990); L. Blanchet and T. Damour, Phys. Rev. D 46, 4304 (1992); C. Cutler et al., Phys. Rev. Lett. 70, 2984 (1993); C. Cutler and E. E. Flanagan, Phys. Rev. D 49, 2658 (1994); T. A. Apostolatos, C. Cutler, G. J. Sussman, and K. S. Thorne, Phys. Rev. D 49, 6274 (1994).

[4] L. E. Kidder, C. M. Will, and A. G. Wiseman, Classical Quantum Gravity 9, L125 (1992); L. E. Kidder, C. M. Will, and A. G. Wiseman, Phys. Rev. D 47, 3281 (1993).

[5] L. Blanchet et al., Phys. Rev. Lett. 74, 14, 3515 (1995); C. M. Will and A. G. Wiseman, Phys. Rev. D 54, 4813 (1996).

[6] C. S. Kochanek, Astrophys. J. 398, 234 (1992).

[7] L. Bildsten and C. Cutler, Astrophys. J. 400, 175 (1992).

[8] D. Lai, F. A. Rasio, and S. L. Shapiro, Astrophys. J. Supp. 88, 205 (1993).

[9] D. Lai, F. A. Rasio, and S. L. Shapiro, Astrophys. J. 406, L63 (1993).

[10] D. Lai, F. A. Rasio, and S. L. Shapiro, Astrophys. J. 420, 811 (1994).

[11] D. Lai, F. A. Rasio, and S. L. Shapiro, Astrophys. J. 423, 344 (1994).

[12] D. Lai, F. A. Rasio, and S. L. Shapiro, Astrophys. J. 437, 742 (1994).

[13] D. Lai and S. L. Shapiro, Astrophys. J. 443, 705 (1995).

[14] K. Oohara and T. Nakamura, Prog. Theor. Phys. 88, 307 (1992); M. Shibata, T. Nakamura, and K. Oohara, ibid. 88, 1079 (1992); Rasio, F. A., and Shapiro, S. L., ApJ, 401, 226 (1992); Rasio, F. A., and Shapiro, S. L., ApJ, 432, 242 (1994); M. B. Davies, W. 
Benz, T. Piran, and F. K. Thielemann, Astrophys. J. 431, 742 (1994); X. Zughe, J. M. Centrella, and S. L. W. McMillan, Phys. Rev. D 50, 6247 (1994); M. Ruffert, H. T. Janka, and G. Schäfer, Astrophys. Sp. Sci. 231, 423 (1995).

[15] For earlier work, see articles in Sources of Gravitational Radiation, edited by L. Smarr (Cambridge Univ. Press, Cambridge, 1979), and in Frontiers in Numerical Relativity, edited by C. R. Evans, L. S. Finn, and D. W. Hobill (Cambridge Univ. Press, Cambridge 1989). For more recent progress, see R. A. Matzner et al., Science 270, 941 (1995).

[16] J. R. Wilson and G. J. Mathews, Phys. Rev. Lett., 75, 23, 4161 (1995); J. R. Wilson, G. J. Mathews, and P. Marronetti, Phys. Rev. D 54, 1317 (1996); G. J. Mathews and J. R. Wilson, (1997).

[17] D. Lai, Phys Rev. Lett. 76, 4878 (1996).

[18] T. Baumgarte et al., submitted to Phys. Rev. D.

[19] G. B. Cook, Phys. Rev. D 50, 5025 (1994).

[20] K. Taniguchi and T. Nakamura, Prog. Theor. Phys. 96, 693 (1996).

[21] D. Lai and A. G. Wiseman, Phys. Rev. D 54, 3958 (1996).

[22] K. Taniguchi and M. Shibata, Phys. Rev. D 56, in press (1997); M. Shibata and K. Taniguchi, Phys. Rev. D 56, in press (1997).

[23] C. Chandrasekhar, Ellipsoidal Figures of Equilibrium, (Yale Univ. Press, New Haven 1969).

[24] C. Chandrasekhar, An Introduction to the Study of Stellar Structure, (Univ. of Chicago Press, Chicago, Illinois, 1939).

[25] T. Damour, in 300 Years of Gravitation, edited by S. Hawking and W. Israel (Cambridge Univ. Press, Cambridge, 1987), p. 128. 
[26] R. B. Wiringa, V. Fiks, and A. Fabrocini, Phys Rev. C 38, 1010 (1988).

[27] W. D. Arnett and R. L. Bowers, Astrophys. J. 33, 415 (1977).

[28] D. M. Eardley and E. W. Hirschmann, gr-qc/9601019 (1996).

[29] M. Shibata, Prog. Theor. Phys. 96, 317 (1996); M. Shibata, Phys. Rev. D 55, 6019 (1997).

[30] S. L. Shapiro and S. A. Teukolsky, Black Holes, White Dwarfs, and Neutron Stars (John Wiley \& Sons, Inc., New York, 1983).

[31] P. R. Brady and S. A. Hughes, gr-qc/9704019 (1997).

[32] A. G. Wiseman, gr-qc/9704018 (1997).

[33] C. Chandrasekhar, Astrophys. J. 142, 1488 (1965).

[34] C. Chandrasekhar and Y. Nutku, Astrophys. J. 158, 55 (1969).

[35] R. F. Tooper, Astrophys. J. 142, 1541 (1965).

[36] Y. B. Zel'dovich and I. D. Novikov, Relativistic Astrophysics (University of Chicago Press, Chicago, Illinois, 1971), Vol. 1. 


\section{TABLES}

TABLE I. The polytropic coefficients to the PN energy corrections of an isolated spherical star, as determined from equations (B42) and (B52).

\begin{tabular}{|c|c|c|}
\hline$n$ & $l_{1}$ & $l_{2}$ \\
\hline 0.0 & 0.000000 & 0.111365 \\
\hline 0.1 & 0.094949 & 0.097502 \\
\hline 0.2 & 0.191634 & 0.084840 \\
\hline 0.3 & 0.289737 & 0.073070 \\
\hline 0.4 & 0.388996 & 0.061974 \\
\hline 0.5 & 0.489198 & 0.051391 \\
\hline 0.6 & 0.590166 & 0.041201 \\
\hline 0.7 & 0.691752 & 0.031312 \\
\hline 0.8 & 0.793835 & 0.021649 \\
\hline 0.9 & 0.896311 & 0.012154 \\
\hline 1.0 & 0.999096 & 0.002779 \\
\hline 1.1 & 1.102118 & -0.006516 \\
\hline 1.2 & 1.205321 & -0.015767 \\
\hline 1.3 & 1.308657 & -0.025003 \\
\hline 1.4 & 1.412088 & -0.034252 \\
\hline 1.5 & 1.515586 & -0.043538 \\
\hline 2.0 & 2.033637 & -0.091306 \\
\hline 2.5 & 2.553451 & -0.143291 \\
\hline 3.0 & 3.080363 & -0.202770 \\
\hline 3.5 & 3.628218 & -0.275041 \\
\hline 4.0 & 4.231742 & -0.371100 \\
\hline 4.5 & 5.003087 & -0.523069 \\
\hline
\end{tabular}


TABLE II. Isolated spherical polytropic models, as calculated from the PN-accurate energy functional (B44). Here $n$ is the polytropic index; $K \rho_{o c}^{1 / n} / c^{2}$ is the central density parameter; $M$ is the gravitational mass; $R$ is the stellar radius in Schwarzschild coordinates as calculated from eq. (5); $M_{o}$ is the rest mass; and $R_{o}$ is the Newtonian radius defined by eq. (44). The value of $K \rho_{o c} / c^{2}$ marked by an asterisk $\left(^{*}\right)$ identifies the star with maximum rest mass $M_{o}$ and gravitational mass $M$ for that value of $n$.

\begin{tabular}{|c|c|c|c|c|c|}
\hline$n$ & $\log K \rho_{o c} / c^{2}$ & $G M / R c^{2}$ & $G M_{o} / R_{o} c^{2}$ & $M / M_{O}$ & $M_{o} /\left(c^{3-n} G^{-3 / 2} K^{n / 2}\right)$ \\
\hline 0.5 & -1.0693 & 0.125 & 0.1234 & 0.9276 & 0.0580 \\
\hline 0.5 & -1.0076 & 0.1380 & 0.1359 & 0.9198 & 0.0655 \\
\hline 0.5 & -0.7687 & 0.1941 & 0.1883 & 0.8853 & 0.0984 \\
\hline 0.5 & -0.7450 & 0.2 & 0.1936 & 0.8816 & 0.1019 \\
\hline 0.5 & -0.5541 & 0.2481 & 0.2348 & 0.8517 & 0.1297 \\
\hline 0.5 & -0.5464 & 0.25 & 0.2364 & 0.8505 & 0.1308 \\
\hline 0.5 & $0.1212^{*}$ & 0.3917 & 0.3058 & 0.7875 & 0.1804 \\
\hline 1.0 & -1.9749 & 0.02 & 0.0196 & 0.9900 & 0.0246 \\
\hline 1.0 & -1.0180 & 0.125 & 0.1074 & 0.9387 & 0.1346 \\
\hline 1.0 & -0.8907 & 0.15 & 0.1232 & 0.9275 & 0.1545 \\
\hline 1.0 & -0.6532 & 0.2 & 0.1467 & 0.9083 & 0.1838 \\
\hline 1.0 & -0.4171 & 0.25 & 0.1563 & 0.8984 & 0.1960 \\
\hline 1.0 & $-0.3902^{*}$ & 0.2556 & 0.1564 & 0.8983 & 0.1961 \\
\hline
\end{tabular}


TABLE III. Irrotational Equilibrium Sequences. The dimensionless quantities $\bar{\Omega}, \bar{J}$, and $\bar{E}$ are defined in equation (43).

\begin{tabular}{|c|c|c|c|c|c|c|c|c|c|}
\hline$r / a_{1}$ & $r / R_{o}$ & $r c^{2} / G m$ & $a_{2} / a_{1}$ & $a_{3} / a_{1}$ & $T_{s} /|W|$ & $\bar{\Omega}$ & $\bar{J}$ & $\bar{E}$ & $R / R_{O}$ \\
\hline \multicolumn{10}{|c|}{$G M / R c^{2}=0$ (Newtonian treatment), $n=0.5$} \\
\hline 6.0 & 6.052 & $\infty$ & 0.9871 & 0.9874 & $0.371(-6)$ & 0.1097 & 1.7401 & -1.1937 & 1.0000 \\
\hline 5.0 & 5.075 & $\infty$ & 0.9776 & 0.9783 & $0.193(-5)$ & 0.1430 & 1.5944 & -1.2095 & 1.0000 \\
\hline 4.0 & 4.120 & $\infty$ & 0.9557 & 0.9579 & $0.146(-4)$ & 0.1958 & 1.4392 & -1.2320 & 1.0002 \\
\hline 3.5 & 3.661 & $\infty$ & 0.9331 & 0.9378 & $0.492(-4)$ & 0.2343 & 1.3602 & -1.2467 & 1.0004 \\
\hline 3.2 & 3.396 & $\infty$ & 0.9117 & 0.9193 & $0.111(-3)$ & 0.2629 & 1.3145 & -1.2565 & 1.0006 \\
\hline 3.0 & 3.228 & $\infty$ & 0.8921 & 0.9027 & $0.200(-3)$ & 0.2846 & 1.2859 & -1.2633 & 1.0009 \\
\hline 2.8 & 3.068 & $\infty$ & 0.8663 & 0.8814 & $0.373(-3)$ & 0.3084 & 1.2603 & -1.2698 & 1.0014 \\
\hline 2.6 & 2.920 & $\infty$ & 0.8322 & 0.8537 & $0.724(-3)$ & 0.3340 & 1.2401 & -1.2754 & 1.0023 \\
\hline $2.344^{*}$ & 2.759 & $\infty$ & 0.7716 & 0.8049 & $0.178(-2)$ & 0.3681 & 1.2283 & -1.2790 & 1.0042 \\
\hline 2.0 & 2.623 & $\infty$ & 0.6498 & 0.7045 & $0.635(-2)$ & 0.4090 & 1.2651 & -1.2663 & 1.0107 \\
\hline \multicolumn{10}{|c|}{$G M / R c^{2}=0.2, n=0.5$} \\
\hline 6.0 & 5.173 & 13.36 & 0.9845 & 0.9848 & $0.424(-6)$ & 0.1146 & 1.5553 & -1.2860 & 0.8533 \\
\hline 5.0 & 4.344 & 11.22 & 0.9732 & 0.9737 & $0.210(-5)$ & 0.1458 & 1.4727 & -1.2953 & 0.8534 \\
\hline 4.0 & 3.537 & 9.14 & 0.9475 & 0.9495 & $0.146(-4)$ & 0.1930 & 1.3956 & -1.3064 & 0.8536 \\
\hline 3.5 & 3.150 & 8.14 & 0.9221 & 0.9259 & $0.457(-4)$ & 0.2260 & 1.3647 & -1.3120 & 0.8539 \\
\hline 3.2 & 2.928 & 7.56 & 0.8989 & 0.9049 & $0.972(-4)$ & 0.2499 & 1.3520 & -1.3146 & 0.8542 \\
\hline 3.0 & 2.787 & 7.20 & 0.8784 & 0.8866 & $0.166(-3)$ & 0.2679 & 1.3477 & -1.3155 & 0.8547 \\
\hline $2.922^{*}$ & 2.734 & 7.06 & 0.8690 & 0.8782 & $0.207(-3)$ & 0.2753 & 1.3473 & -1.3156 & 0.8549 \\
\hline 2.8 & 2.652 & 6.85 & 0.8524 & 0.8636 & $0.293(-3)$ & 0.2875 & 1.3485 & -1.3153 & 0.8553 \\
\hline 2.6 & 2.527 & 6.53 & 0.8192 & 0.8345 & $0.534(-3)$ & 0.3090 & 1.3566 & -1.3132 & 0.8563 \\
\hline 2.0 & 2.258 & 5.83 & 0.6525 & 0.6899 & $0.396(-2)$ & 0.3794 & 1.4748 & -1.2770 & 0.8653 \\
\hline 1.736 & 2.245 & 5.80 & 0.5351 & 0.5835 & $0.103(-1)$ & 0.4036 & 1.6258 & -1.2255 & 0.8772 \\
\hline \multicolumn{10}{|c|}{$G M / R c^{2}=0.25, n=0.5$} \\
\hline 6.0 & 4.878 & 10.32 & 0.9836 & 0.9838 & $0.437(-6)$ & 0.1171 & 1.4952 & -1.3223 & 0.8042 \\
\hline 5.0 & 4.099 & 8.67 & 0.9716 & 0.9721 & $0.213(-5)$ & 0.1479 & 1.4296 & -1.3297 & 0.8043 \\
\hline 4.0 & 3.340 & 7.07 & 0.9446 & 0.9465 & $0.144(-4)$ & 0.1938 & 1.3749 & -1.3377 & 0.8045 \\
\hline 3.5 & 2.978 & 6.30 & 0.9182 & 0.9218 & $0.444(-4)$ & 0.2256 & 1.3588 & -1.3406 & 0.8048 \\
\hline $3.280^{*}$ & 2.825 & 5.98 & 0.9014 & 0.9064 & $0.760(-4)$ & 0.2421 & 1.3566 & -1.3410 & 0.8051 \\
\hline 3.2 & 2.770 & 5.86 & 0.8943 & 0.8998 & $0.931(-4)$ & 0.2486 & 1.3570 & -1.3409 & 0.8053 \\
\hline 3.0 & 2.638 & 5.58 & 0.8733 & 0.8808 & $0.157(-3)$ & 0.2658 & 1.3612 & -1.3400 & 0.8057 \\
\hline 2.8 & 2.512 & 5.31 & 0.8470 & 0.8571 & $0.274(-3)$ & 0.2847 & 1.3717 & -1.3375 & 0.8065 \\
\hline 2.6 & 2.396 & 5.07 & 0.8136 & 0.8274 & $0.493(-3)$ & 0.3053 & 1.3913 & -1.3324 & 0.8076 \\
\hline 2.0 & 2.145 & 4.54 & 0.6490 & 0.6822 & $0.352(-2)$ & 0.3748 & 1.5575 & -1.2825 & 0.8174 \\
\hline 1.682 & 2.146 & 4.54 & 0.5069 & 0.5517 & $0.112(-1)$ & 0.4034 & 1.7850 & -1.2052 & 0.8341 \\
\hline
\end{tabular}




\begin{tabular}{|c|c|c|c|c|c|c|c|c|c|}
\hline \multicolumn{10}{|c|}{$G M / R c^{2}=0.2, n=1.0$} \\
\hline 6.0 & 4.026 & 13.72 & 0.9894 & 0.9895 & $0.144(-6)$ & 0.1713 & 1.4318 & -1.3369 & 0.6663 \\
\hline 5.0 & 3.373 & 11.50 & 0.9816 & 0.9819 & $0.719(-6)$ & 0.2188 & 1.3528 & -1.3502 & 0.6663 \\
\hline 4.0 & 2.732 & 9.31 & 0.9638 & 0.9648 & $0.506(-5)$ & 0.2917 & 1.2766 & -1.3668 & 0.6667 \\
\hline 3.5 & 2.422 & 8.25 & 0.9459 & 0.9480 & $0.161(-4)$ & 0.3434 & 1.2431 & -1.3759 & 0.6672 \\
\hline 3.2 & 2.241 & 7.64 & 0.9294 & 0.9327 & $0.346(-4)$ & 0.3813 & 1.2266 & -1.3811 & 0.6679 \\
\hline 3.0 & 2.125 & 7.24 & 0.9147 & 0.9193 & $0.598(-4)$ & 0.4098 & 1.2182 & -1.3840 & 0.6686 \\
\hline 2.8 & 2.013 & 6.86 & 0.8957 & 0.9022 & $0.107(-3)$ & 0.4413 & 1.2127 & -1.3860 & 0.6698 \\
\hline $2.660^{*}$ & 1.939 & 6.61 & 0.8792 & 0.8874 & $0.163(-3)$ & 0.4651 & 1.2115 & -1.3865 & 0.6710 \\
\hline 2.6 & 1.908 & 6.50 & 0.8712 & 0.8803 & $0.197(-3)$ & 0.4757 & 1.2117 & -1.3864 & 0.6717 \\
\hline 2.0 & 1.664 & 5.67 & 0.7405 & 0.7667 & $0.159(-2)$ & 0.5884 & 1.2636 & -1.3615 & 0.6889 \\
\hline 1.721 & 1.636 & 5.58 & 0.6317 & 0.6720 & $0.481(-2)$ & 0.6266 & 1.3521 & -1.3146 & 0.7143 \\
\hline \multicolumn{10}{|c|}{$G M / R c^{2}=0.25, n=1.0$} \\
\hline 6.0 & 3.398 & 10.87 & 0.9890 & 0.9891 & $0.149(-6)$ & 0.2128 & 1.3490 & -1.3952 & 0.5621 \\
\hline 5.0 & 2.850 & 9.11 & 0.9808 & 0.9811 & $0.739(-6)$ & 0.2700 & 1.2860 & -1.4083 & 0.5626 \\
\hline 4.0 & 2.316 & 7.41 & 0.9623 & 0.9633 & $0.512(-5)$ & 0.3557 & 1.2303 & -1.4232 & 0.5647 \\
\hline 3.5 & 2.063 & 6.60 & 0.9438 & 0.9458 & $0.160(-4)$ & 0.4144 & 1.2099 & -1.4299 & 0.5675 \\
\hline 3.2 & 1.919 & 6.14 & 0.9269 & 0.9301 & $0.342(-4)$ & 0.4559 & 1.2024 & -1.4327 & 0.5706 \\
\hline 3.0 & 1.827 & 5.84 & 0.9118 & 0.9162 & $0.587(-4)$ & 0.4863 & 1.2006 & -1.4334 & 0.5737 \\
\hline $2.987^{*}$ & 1.822 & 5.83 & 0.9107 & 0.9152 & $0.608(-4)$ & 0.4884 & 1.2006 & -1.4335 & 0.5739 \\
\hline 2.8 & 1.741 & 5.57 & 0.8926 & 0.8987 & $0.104(-3)$ & 0.5189 & 1.2023 & -1.4327 & 0.5779 \\
\hline 2.6 & 1.662 & 5.32 & 0.8678 & 0.8765 & $0.190(-3)$ & 0.5531 & 1.2089 & -1.4296 & 0.5836 \\
\hline 2.0 & 1.500 & 4.80 & 0.7382 & 0.7628 & $0.149(-2)$ & 0.6538 & 1.2835 & -1.3897 & 0.6194 \\
\hline 1.688 & 1.514 & 4.84 & 0.6158 & 0.6556 & $0.515(-2)$ & 0.6787 & 1.3948 & -1.3250 & 0.6631 \\
\hline
\end{tabular}


TABLE IV. Irrotational Equilibrium Sequences: Energy Terms

\begin{tabular}{|c|c|c|c|c|c|c|c|c|c|c|}
\hline \multicolumn{10}{|c|}{$G M / R c^{2}=0.2, n=1.0$} & $\bar{E}$ \\
\hline 6.0 & 1.5147 & -2.1704 & 0.0000 & 0.0000 & -0.1234 & -0.0010 & -0.5927 & -0.0015 & 0.0374 & -1.3369 \\
\hline 5.0 & 1.5142 & -2.1701 & 0.0000 & -0.0001 & -0.1440 & -0.0012 & -0.5924 & -0.0015 & 0.0450 & -1.3502 \\
\hline 4.0 & 1.5121 & -2.1689 & 0.0000 & -0.0005 & -0.1714 & -0.0015 & -0.5913 & -0.0015 & 0.0562 & -1.3668 \\
\hline 3.5 & 1.5086 & -2.1669 & 0.0000 & -0.0010 & -0.1879 & -0.0016 & -0.5895 & -0.0014 & 0.0638 & -1.3759 \\
\hline 3.2 & 1.5042 & -2.1644 & 0.0001 & -0.0016 & -0.1981 & -0.0017 & -0.5872 & -0.0014 & 0.0692 & -1.3811 \\
\hline 3.0 & 1.4993 & -2.1616 & 0.0001 & -0.0023 & -0.2047 & -0.0018 & -0.5847 & -0.0014 & 0.0731 & -1.3840 \\
\hline 2.8 & 1.4917 & -2.1572 & 0.0002 & -0.0034 & -0.2106 & -0.0018 & -0.5807 & -0.0014 & 0.0774 & -1.3860 \\
\hline $2.660^{*}$ & 1.4839 & -2.1526 & 0.0004 & -0.0045 & -0.2140 & -0.0019 & -0.5767 & -0.0014 & 0.0804 & -1.3865 \\
\hline 2.6 & 1.4797 & -2.1502 & 0.0004 & -0.0051 & -0.2151 & -0.0019 & -0.5745 & -0.0014 & 0.0817 & -1.3864 \\
\hline 2.0 & 1.3752 & -2.0864 & 0.0033 & -0.0182 & -0.2027 & -0.0027 & -0.5211 & -0.0014 & 0.0925 & -1.3615 \\
\hline 1.696 & 1.2219 & -1.9844 & 0.0106 & -0.0349 & -0.1615 & -0.0041 & -0.4451 & -0.0013 & 0.0906 & -1.3082 \\
\hline
\end{tabular}

TABLE V. Irrotational Equilibrium Sequences: Angular Momentum Terms

\begin{tabular}{cccccc}
\hline \hline$r / a_{1}$ & $\bar{J}_{H}$ & $\bar{J}_{s}+\bar{J}_{s}^{\prime}$ & $\bar{J}_{P N}$ & $\bar{J}_{O I}+\bar{J}_{O I}^{\prime}$ & \\
& & $G M / R c^{2}=0.2, n=1.0$ & \\
\hline 6.0 & 1.5437 & 0.0000 & -0.0017 & -0.1102 & 1.4318 \\
5.0 & 1.4524 & 0.0000 & -0.0008 & -0.0988 & 1.3528 \\
4.0 & 1.3623 & 0.0001 & 0.0006 & -0.0864 & 1.2766 \\
3.5 & 1.3213 & 0.0002 & 0.0015 & -0.0799 & 1.2431 \\
3.2 & 1.3000 & 0.0005 & 0.0022 & -0.0760 & 1.2266 \\
3.0 & 1.2882 & 0.0007 & 0.0026 & -0.0734 & 1.2182 \\
2.8 & 1.2794 & 0.0012 & 0.0030 & -0.0709 & 1.2127 \\
$2.660^{*}$ & 1.2757 & 0.0017 & 0.0032 & -0.0692 & 1.2115 \\
2.6 & 1.2749 & 0.0021 & 0.0033 & -0.0685 & 1.2117 \\
2.0 & 1.3112 & 0.0130 & 0.0032 & -0.0638 & 1.2636 \\
1.696 & 1.3890 & 0.0388 & 0.0008 & -0.0647 & 1.3638 \\
\hline \hline
\end{tabular}


TABLE VI. Equilibrium Corotational Sequences. The dimensionless quantities $\bar{\Omega}, \bar{J}, \bar{E}$, and $\overline{\mathcal{C}}$ are defined in equation (43).

\begin{tabular}{|c|c|c|c|c|c|c|c|c|c|c|}
\hline$r / a_{1}$ & $r / R_{o}$ & $r c^{2} / G m$ & $a_{2} / a_{1}$ & $a_{3} / a_{1}$ & $T_{s} /|W|$ & $\bar{\Omega}$ & $\bar{J}$ & $\bar{E}$ & $R / R_{o}$ & $\overline{\mathcal{C}}$ \\
\hline \multicolumn{11}{|c|}{$G M / R c^{2}=0$ (Newtonian treatment), $n=0.5$} \\
\hline 6.0 & 6.073 & $\infty$ & 0.9874 & 0.9794 & $0.221(-2)$ & 0.1092 & 1.8057 & -1.1904 & 1.0009 & -0.0623 \\
\hline 5.0 & 5.105 & $\infty$ & 0.9784 & 0.9651 & $0.376(-2)$ & 0.1418 & 1.6809 & -1.2039 & 1.0016 & -0.0816 \\
\hline 4.0 & 4.162 & $\infty$ & 0.9585 & 0.9348 & $0.711(-2)$ & 0.1929 & 1.5603 & -1.2211 & 1.0031 & -0.1130 \\
\hline 3.5 & 3.710 & $\infty$ & 0.9390 & 0.9065 & $0.103(-1)$ & 0.2299 & 1.5072 & -1.2307 & 1.0046 & -0.1369 \\
\hline 3.2 & 3.450 & $\infty$ & 0.9214 & 0.8819 & $0.131(-1)$ & 0.2571 & 1.4810 & -1.2363 & 1.0061 & -0.1554 \\
\hline 3.0 & 3.283 & $\infty$ & 0.9058 & 0.8609 & $0.156(-1)$ & 0.2777 & 1.4674 & -1.2394 & 1.0074 & -0.1701 \\
\hline 2.8 & 3.124 & $\infty$ & 0.8860 & 0.8352 & $0.186(-1)$ & 0.3005 & 1.4583 & -1.2417 & 1.0092 & -0.1871 \\
\hline $2.625^{*}$ & 2.992 & $\infty$ & 0.8641 & 0.8079 & $0.219(-1)$ & 0.3221 & 1.4553 & -1.2425 & 1.0112 & -0.2042 \\
\hline 2.6 & 2.974 & $\infty$ & 0.8605 & 0.8035 & $0.224(-1)$ & 0.3254 & 1.4554 & -1.2424 & 1.0115 & -0.2068 \\
\hline $2.115^{* *}$ & 2.677 & $\infty$ & 0.7609 & 0.6909 & $0.367(-1)$ & 0.3915 & 1.4949 & -1.2298 & 1.0218 & -0.2696 \\
\hline 2.0 & 2.630 & $\infty$ & 0.7256 & 0.6543 & $0.416(-1)$ & 0.4066 & 1.5208 & -1.2208 & 1.0259 & -0.2880 \\
\hline \multicolumn{11}{|c|}{$G M / R c^{2}=0.2, n=0.5$} \\
\hline 6.0 & 5.189 & 13.40 & 0.9848 & 0.9782 & $0.175(-2)$ & 0.1141 & 1.6096 & -1.2832 & 0.8541 & -0.0524 \\
\hline 5.0 & 4.365 & 11.27 & 0.9739 & 0.9634 & $0.284(-2)$ & 0.1449 & 1.5423 & -1.2907 & 0.8546 & -0.0671 \\
\hline 4.0 & 3.565 & 9.21 & 0.9499 & 0.9324 & $0.503(-2)$ & 0.1911 & 1.4893 & -1.2983 & 0.8558 & -0.0899 \\
\hline 3.5 & 3.182 & 8.22 & 0.9269 & 0.9039 & $0.699(-2)$ & 0.2232 & 1.4756 & -1.3007 & 0.8571 & -0.1067 \\
\hline $3.345^{*}$ & 3.067 & 7.92 & 0.9172 & 0.8920 & $0.780(-2)$ & 0.2348 & 1.4746 & -1.3009 & 0.8576 & -0.1129 \\
\hline 3.2 & 2.962 & 7.65 & 0.9065 & 0.8792 & $0.867(-2)$ & 0.2465 & 1.4756 & -1.3007 & 0.8582 & -0.1194 \\
\hline 3.0 & 2.821 & 7.29 & 0.8887 & 0.8584 & $0.101(-1)$ & 0.2640 & 1.4809 & -1.2995 & 0.8593 & -0.1294 \\
\hline $2.842^{* *}$ & 2.714 & 7.01 & 0.8716 & 0.8387 & $0.115(-1)$ & 0.2790 & 1.4893 & -1.2975 & 0.8604 & -0.1383 \\
\hline 2.8 & 2.687 & 6.94 & 0.8666 & 0.8329 & $0.119(-1)$ & 0.2832 & 1.4922 & -1.2968 & 0.8608 & -0.1408 \\
\hline 2.6 & 2.560 & 6.61 & 0.8388 & 0.8017 & $0.141(-1)$ & 0.3042 & 1.5117 & -1.2918 & 0.8627 & -0.1542 \\
\hline 1.734 & 2.231 & 5.76 & 0.5976 & 0.5539 & $0.357(-1)$ & 0.4054 & 1.8183 & -1.1935 & 0.8899 & -0.2491 \\
\hline \multicolumn{11}{|c|}{$G M / R c^{2}=0.25, n=0.5$} \\
\hline 6.0 & 4.892 & 10.35 & 0.9839 & 0.9778 & $0.160(-2)$ & 0.1167 & 1.5459 & -1.3196 & 0.8050 & -0.0492 \\
\hline 5.0 & 4.117 & 8.71 & 0.9723 & 0.9627 & $0.257(-2)$ & 0.1470 & 1.4941 & -1.3255 & 0.8055 & -0.0625 \\
\hline 4.0 & 3.365 & 7.12 & 0.9470 & 0.9312 & $0.447(-2)$ & 0.1921 & 1.4606 & -1.3303 & 0.8066 & -0.0830 \\
\hline $3.706^{*}$ & 3.151 & 6.67 & 0.9341 & 0.9157 & $0.537(-2)$ & 0.2096 & 1.4580 & -1.3307 & 0.8073 & -0.0912 \\
\hline 3.5 & 3.006 & 6.36 & 0.9227 & 0.9022 & $0.615(-2)$ & 0.2232 & 1.4596 & -1.3304 & 0.8078 & -0.0979 \\
\hline $3.230^{* *}$ & 2.820 & 5.96 & 0.9038 & 0.8801 & $0.741(-2)$ & 0.2432 & 1.4674 & -1.3288 & 0.8089 & -0.1079 \\
\hline 3.2 & 2.800 & 5.92 & 0.9013 & 0.8773 & $0.757(-2)$ & 0.2456 & 1.4688 & -1.3285 & 0.8090 & -0.1091 \\
\hline 3.0 & 2.668 & 5.64 & 0.8828 & 0.8562 & $0.878(-2)$ & 0.2624 & 1.4813 & -1.3258 & 0.8101 & -0.1180 \\
\hline 2.8 & 2.542 & 5.38 & 0.8598 & 0.8305 & $0.103(-1)$ & 0.2809 & 1.5010 & -1.3211 & 0.8116 & -0.1282 \\
\hline 2.6 & 2.425 & 5.13 & 0.8312 & 0.7990 & $0.122(-1)$ & 0.3012 & 1.5304 & -1.3137 & 0.8136 & -0.1401 \\
\hline 1.679 & 2.131 & 4.51 & 0.5634 & 0.5264 & $0.338(-1)$ & 0.4053 & 1.9611 & -1.1760 & 0.8463 & -0.2354 \\
\hline
\end{tabular}




\begin{tabular}{|c|c|c|c|c|c|c|c|c|c|c|}
\hline \multicolumn{11}{|c|}{$G M / R c^{2}=0.2, n=1.0$} \\
\hline 6.0 & 4.045 & 13.79 & 0.9895 & 0.9847 & $0.127(-2)$ & 0.1702 & 1.4714 & -1.3338 & 0.6683 & -0.0373 \\
\hline 5.0 & 3.398 & 11.58 & 0.9820 & 0.9741 & $0.208(-2)$ & 0.2166 & 1.4040 & -1.3450 & 0.6697 & -0.0479 \\
\hline 4.0 & 2.769 & 9.44 & 0.9651 & 0.9516 & $0.373(-2)$ & 0.2867 & 1.3461 & -1.3574 & 0.6728 & -0.0645 \\
\hline 3.5 & 2.466 & 8.41 & 0.9486 & 0.9303 & $0.523(-2)$ & 0.3354 & 1.3260 & -1.3628 & 0.6759 & -0.0769 \\
\hline 3.2 & 2.292 & 7.81 & 0.9337 & 0.9117 & $0.652(-2)$ & 0.3706 & 1.3195 & -1.3647 & 0.6787 & -0.0863 \\
\hline $3.056^{*}$ & 2.211 & 7.53 & 0.9246 & 0.9005 & $0.729(-2)$ & 0.3893 & 1.3186 & -1.3650 & 0.6805 & -0.0916 \\
\hline 3.0 & 2.180 & 7.43 & 0.9206 & 0.8957 & $0.762(-2)$ & 0.3969 & 1.3188 & -1.3650 & 0.6813 & -0.0938 \\
\hline 2.8 & 2.073 & 7.06 & 0.9041 & 0.8759 & $0.899(-2)$ & 0.4255 & 1.3220 & -1.3638 & 0.6848 & -0.1025 \\
\hline 2.6 & 1.972 & 6.72 & 0.8831 & 0.8512 & $0.107(-1)$ & 0.4565 & 1.3307 & -1.3605 & 0.6895 & -0.1126 \\
\hline $2.570^{* *}$ & 1.957 & 6.67 & 0.8795 & 0.8470 & $0.110(-1)$ & 0.4613 & 1.3326 & -1.3597 & 0.6903 & -0.1143 \\
\hline 1.730 & 1.703 & 5.81 & 0.6886 & 0.6420 & $0.276(-1)$ & 0.5933 & 1.5182 & -1.2710 & 0.7500 & -0.1913 \\
\hline \multicolumn{11}{|c|}{$G M / R c^{2}=0.25, n=1.0$} \\
\hline 6.0 & 3.460 & 11.06 & 0.9891 & 0.9845 & $0.122(-2)$ & 0.2077 & 1.3903 & -1.3908 & 0.5715 & -0.0339 \\
\hline 5.0 & 2.926 & 9.36 & 0.9813 & 0.9738 & $0.197(-2)$ & 0.2607 & 1.3385 & -1.4012 & 0.5763 & -0.0435 \\
\hline 4.0 & 2.409 & 7.70 & 0.9638 & 0.9510 & $0.350(-2)$ & 0.3381 & 1.2990 & -1.4113 & 0.5849 & -0.0585 \\
\hline 3.5 & 2.163 & 6.92 & 0.9468 & 0.9296 & $0.487(-2)$ & 0.3901 & 1.2895 & -1.4142 & 0.5921 & -0.0698 \\
\hline $3.371^{*}$ & 2.101 & 6.72 & 0.9408 & 0.9222 & $0.534(-2)$ & 0.4054 & 1.2890 & -1.4144 & 0.5945 & -0.0733 \\
\hline 3.2 & 2.022 & 6.47 & 0.9315 & 0.9108 & $0.606(-2)$ & 0.4267 & 1.2900 & -1.4140 & 0.5982 & -0.0784 \\
\hline 3.0 & 1.933 & 6.18 & 0.9181 & 0.8947 & $0.707(-2)$ & 0.4534 & 1.2942 & -1.4124 & 0.6033 & -0.0853 \\
\hline $2.839^{* *}$ & 1.864 & 5.96 & 0.9049 & 0.8791 & $0.805(-2)$ & 0.4763 & 1.3006 & -1.4098 & 0.6083 & -0.0917 \\
\hline 2.8 & 1.848 & 5.91 & 0.9013 & 0.8749 & $0.832(-2)$ & 0.4820 & 1.3026 & -1.4090 & 0.6097 & -0.0934 \\
\hline 2.6 & 1.769 & 5.66 & 0.8799 & 0.8502 & $0.990(-2)$ & 0.5123 & 1.3167 & -1.4029 & 0.6176 & -0.1029 \\
\hline 1.700 & 1.596 & 5.10 & 0.6739 & 0.6301 & $0.269(-1)$ & 0.6335 & 1.5440 & -1.2852 & 0.7053 & -0.1832 \\
\hline
\end{tabular}


TABLE VII. Physical quantities at the ISCO for selected irrotational sequences. Here $M_{1.4}=M /\left(1.4 M_{\odot}\right)$ and $M_{1.6}^{\max }=M^{\max } /\left(1.6 M_{\odot}\right)$, where $M^{\max }$ is the maximum value of the isolated gravitational mass $M$.

\begin{tabular}{|c|c|c|c|c|c|c|c|c|c|c|c|}
\hline \multirow[b]{2}{*}{$n$} & \multirow[b]{2}{*}{$G M / R c^{2}$} & \multirow[b]{2}{*}{$G M / R_{o} c^{2}$} & \multirow[b]{2}{*}{$J c / G M_{o}^{2}$} & \multirow[b]{2}{*}{$M_{t}^{2} / M_{o}^{2}$} & \multirow[b]{2}{*}{$a_{2} / a_{1}$} & \multirow[b]{2}{*}{$a_{3} / a_{1}$} & \multirow[b]{2}{*}{$\begin{array}{c}M_{1.4} f_{I S C O} \\
{[\mathrm{~Hz}]}\end{array}$} & \multirow[b]{2}{*}{$\begin{array}{c}M_{1.6}^{\max } f_{I S C O} \\
{[\mathrm{~Hz}]} \\
\end{array}$} & \multicolumn{3}{|c|}{ Ref. 21]'s Results } \\
\hline & & & & & & & & & $a_{2} / a_{1}$ & $a_{3} / a_{1}$ & $\begin{array}{c}M_{1.4} f_{I S C O} \\
{[\mathrm{~Hz}]} \\
\end{array}$ \\
\hline 0.5 & 0.125 & 0.1145 & 3.7178 & 3.387 & 0.821 & 0.839 & 258 & 596 & & & \\
\hline 0.5 & 0.1380 & 0.125 & 3.5656 & 3.326 & 0.828 & 0.845 & 289 & 597 & 0.830 & 0.850 & 279 \\
\hline 0.5 & 0.1941 & 0.1667 & 3.0998 & 3.072 & 0.865 & 0.875 & 404 & 576 & 0.857 & 0.871 & 399 \\
\hline 0.5 & 0.2 & 0.1707 & 3.0622 & 3.046 & 0.869 & 0.878 & 413 & 572 & & & \\
\hline 0.5 & 0.2481 & 0.2 & 2.7997 & 2.841 & 0.900 & 0.905 & 471 & 531 & 0.880 & 0.891 & 488 \\
\hline 0.5 & 0.25 & 0.2010 & 2.7904 & 2.833 & 0.901 & 0.906 & 473 & 529 & & & \\
\hline 1.0 & 0.125 & 0.1008 & 3.6758 & 3.463 & 0.829 & 0.846 & 297 & 362 & & & \\
\hline 1.0 & 0.2 & 0.1332 & 3.1631 & 3.228 & 0.878 & 0.887 & 474 & 438 & & & \\
\hline 1.0 & 0.25 & 0.1405 & 3.0364 & 3.154 & 0.911 & 0.915 & 542 & 475 & & & \\
\hline
\end{tabular}

TABLE VIII. Orbital frequency at the ISCO for selected corotating sequences.

\begin{tabular}{cccccc}
\hline \hline$n$ & $G M / R c^{2}$ & $M_{1.4} f_{I S C O}^{\mathrm{sec}}[\mathrm{Hz}]$ & $M_{1.4} f_{I S C O}^{\mathrm{dyn}}[\mathrm{Hz}]$ & $M_{1.6}^{\max } f_{I S C O}^{\mathrm{sec}}[\mathrm{Hz}]$ & $M_{1.6}^{\max } f_{I S C O}^{\mathrm{dyn}}[\mathrm{Hz}]$ \\
\hline 0.5 & 0.125 & 220 & 267 & 508 & 617 \\
0.5 & 0.2 & 353 & 419 & 488 & 580 \\
0.5 & 0.25 & 409 & 475 & 458 & 531 \\
1.0 & 0.125 & 248 & & & \\
1.0 & 0.2 & 397 & 300 & 302 & 366 \\
1.0 & 0.25 & 450 & 471 & 367 & 434 \\
\hline \hline
\end{tabular}




\section{FIGURES}

FIG. 1. We plot (a) the rest mass $M_{o}$ and (b) the gravitational mass $M=M_{o}+E / c^{2}$ for isolated spherical stars as a function of the central density parameter $K \rho_{o c}^{1 / n} / c^{2}$. Here mass is in units $c^{3-n} G^{-3 / 2} K^{n / 2}$. The solid curve was obtained by integrating the OV equation without approximation. The remaining curves were found by solving the equilibrium equation $0=d E / d \rho_{o c}$ for various energy functionals $E$. The dotted-and-dashed curve results from the use of equation (B47) as the energy functional. The remaining three curves use different values of the coefficients $l_{1}$ and $l_{2}$, via equation ( $(\mathrm{B} 44)$ : The dotted curve is the purely Newtonian result $\left(l_{1}=l_{2}=0\right)$; the short-dashed curve uses the coefficients from equations (B17) and (B18); the long-dashed curve uses our standard coefficients from equations (B42) and (B52).

FIG. 2. The total energy $E$, relative to its value $E_{\infty}$ at infinite separation, as a function of the orbital frequency $f=\Omega / 2 \pi$ for selected $n=0.5$ irrotational sequences. The thick solid curves represent our PN sequences with various $G M / R c^{2}$, where $M$ and $R$ are the isolated neutron star gravitational mass and radius in Schwarzschild coordinates, respectively. The dashed curves represent purely Newtonian results, with the thin curve corresponding to the point mass sequence and the thick curve corresponding to the EFE treatment. The minima of these curves mark the ISCO, inside of which the orbit is dynamically unstable. The sequences terminate when the stars touch.

FIG. 3. Same as Fig. 2, but for an $n=1$ irrotational sequence.

FIG. 4. The dependence of $G M / R c^{2}$ (as calculated in isolation) on the orbital frequency $f_{I S C O}$ at the ISCO for $n=0.5$ and $n=1$ irrotational sequences. For each $n$, the curves are terminated at the value $G M / R c^{2}$ corresponding to the maximum mass.

FIG. 5. Same as Fig. 2, but for an $n=0.5$ corotating sequence. The minima of these curves mark the onset of the secular instability in the orbit. 
FIG. 6. Same as Fig. 5, but for an $n=1$ corotating sequence.

FIG. 7. The rest mass $M_{o}$, normalized to the maximum rest mass of a star at infinite separation $M_{o}^{\infty, \max }$, as a function of the central density parameter $q_{c}=K \rho_{o c}^{1 / n} / c^{2}$ for an $n=1$ corotating binary system and at three different values of the orbital angular frequency $f: f=0$ (solid curve), $M_{1.6}^{\max } f=434 \mathrm{~Hz}$ (long dashed curve), and $M_{1.6}^{\max } f=463 \mathrm{~Hz}$ (short dashed curve). The dotted continuations of the dashed curves represent those binaries inside the innermost dynamically stable orbit.

FIG. 8. The maximum equilibrium rest mass $M_{o}^{\max }$ as a function of the orbital angular frequency $f$ for corotating (dashed curves) and irrotational (solid curves) sequences. The curves are terminated at the values of $f$ for which stars of rest mass $M_{o}=M_{o}^{\infty, \text { max }}$ acquire a dynamically unstable orbit.

FIG. 9. As in Fig. 6, we plot the binding energy versus orbital frequency $f$ for various $n=1$ corotating sequences: $\log q_{c}=-1.15$ (solid curve), -0.87 (dashed curve), and -0.59 (dotted curve). The data points represented by crosses are from the computations of Baumgarte et al., for the same values of $q_{c}$. 
Fig. 1(a)

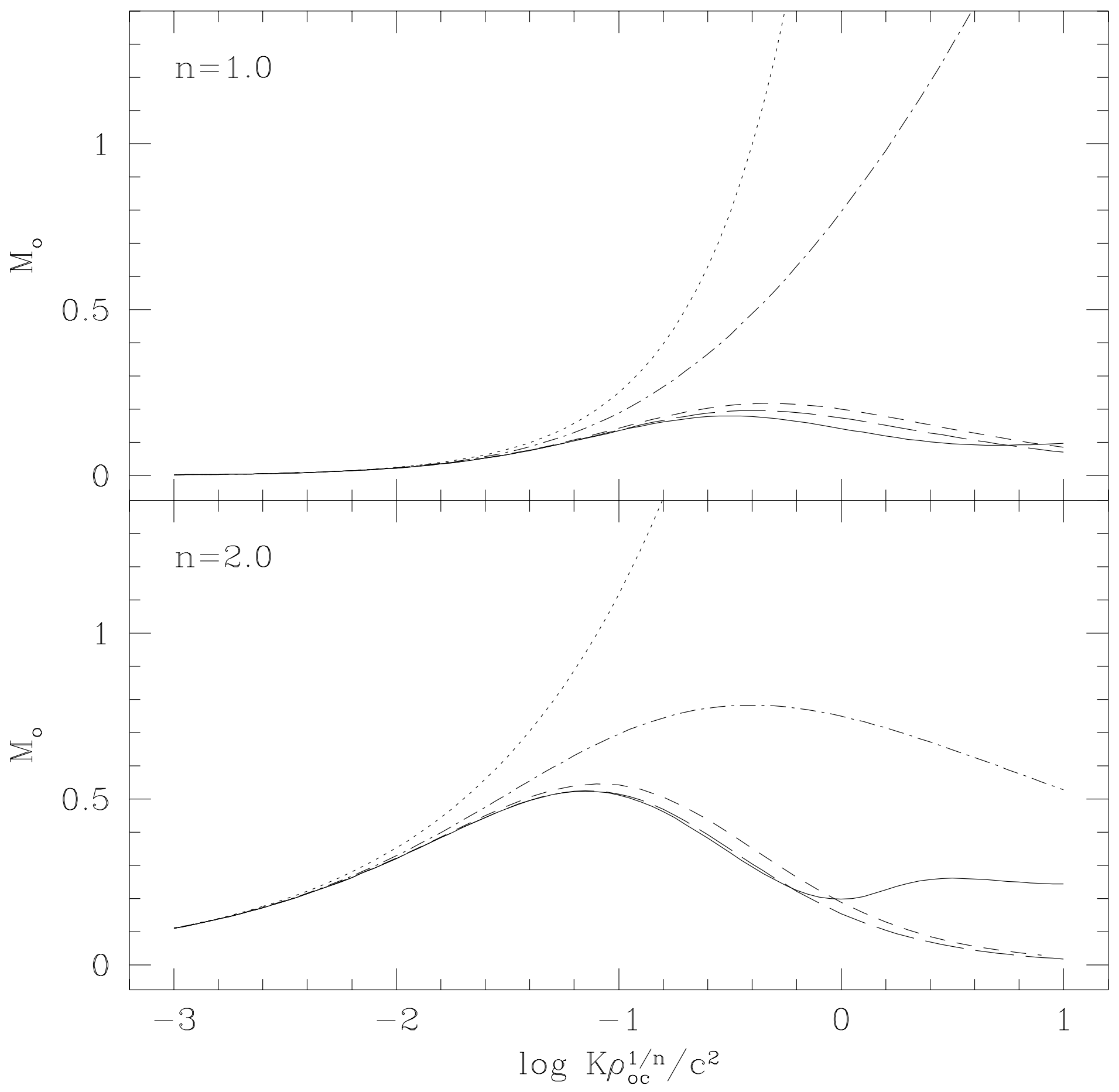


Fig. 1(b)

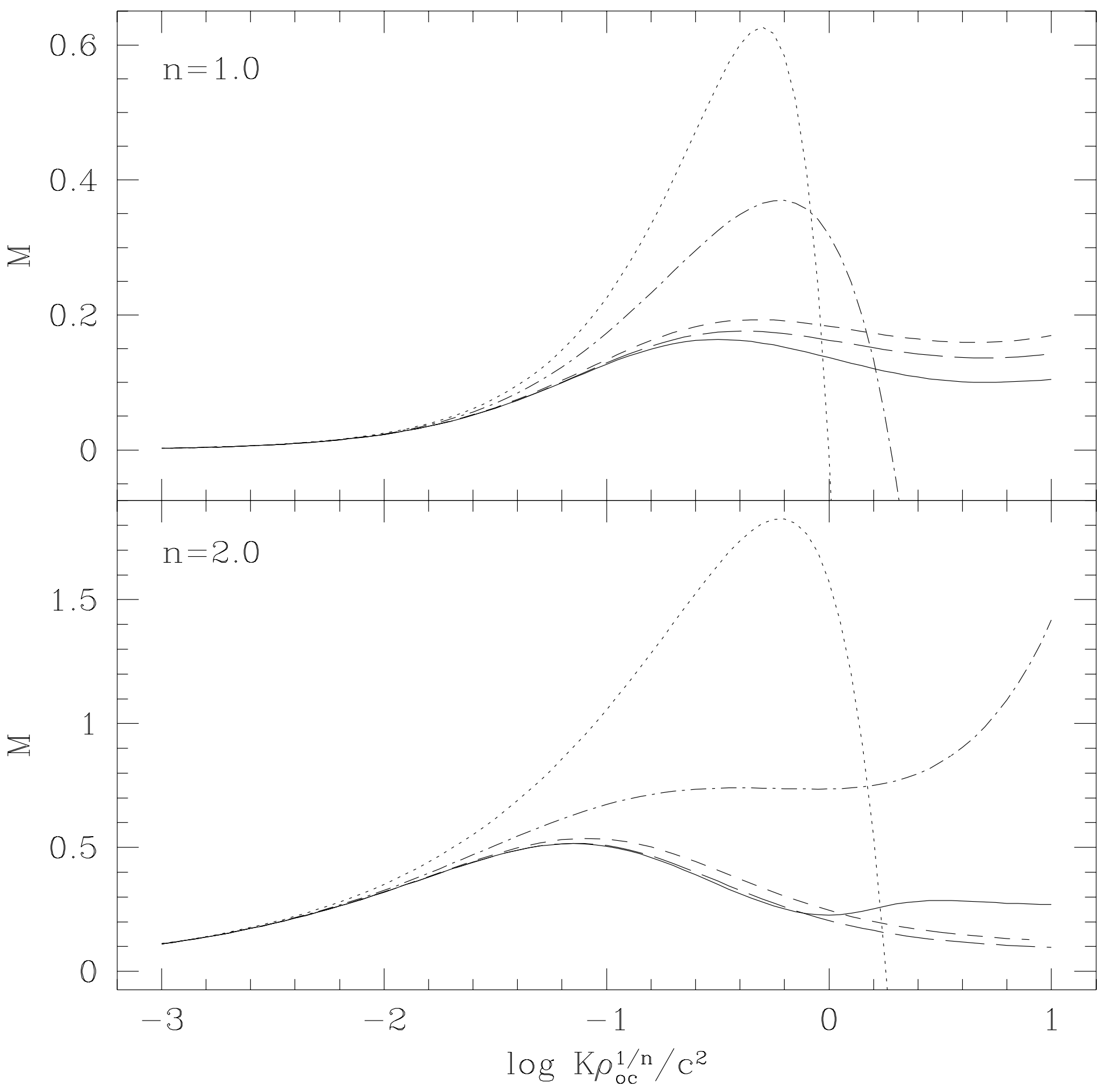




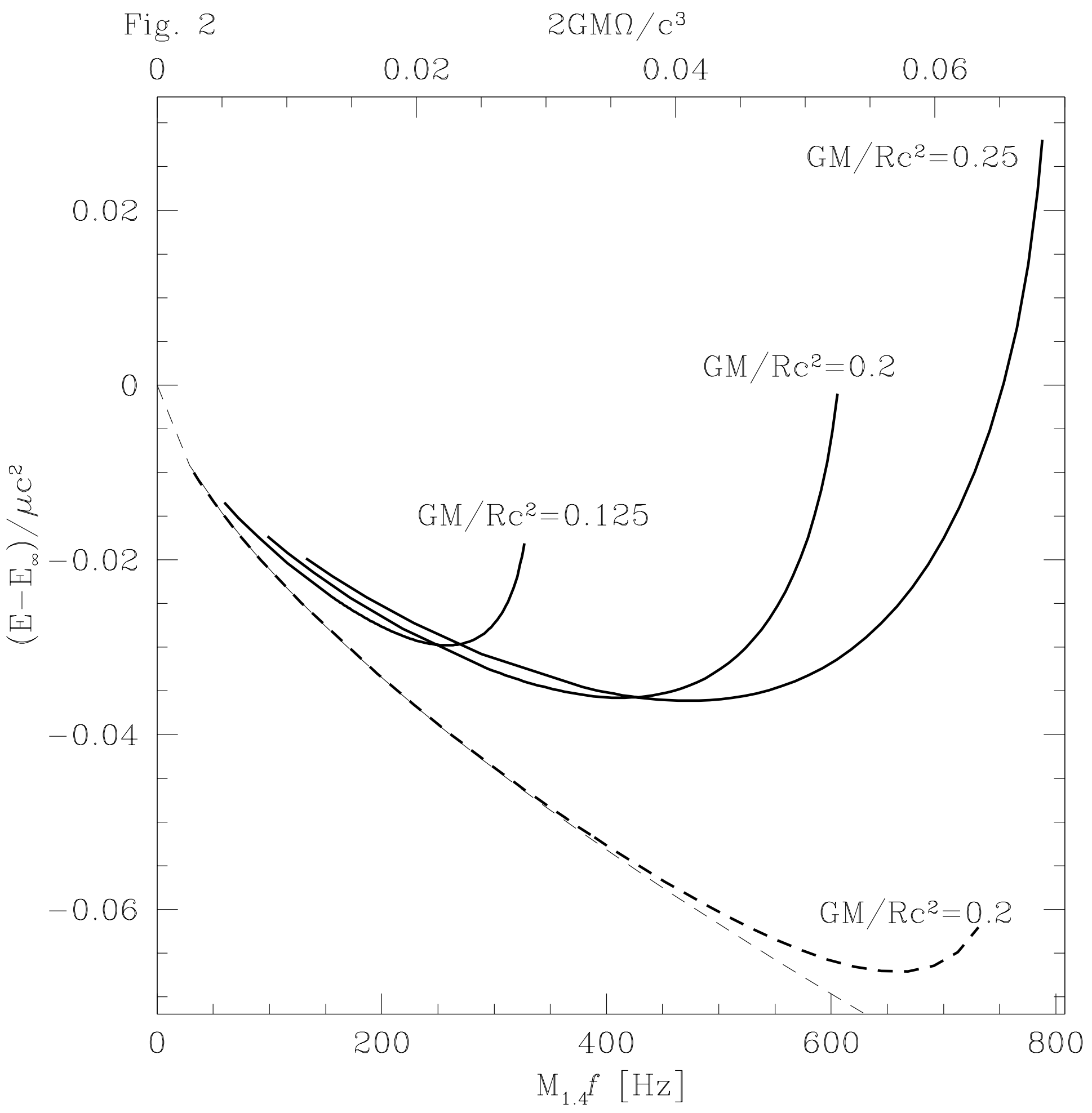




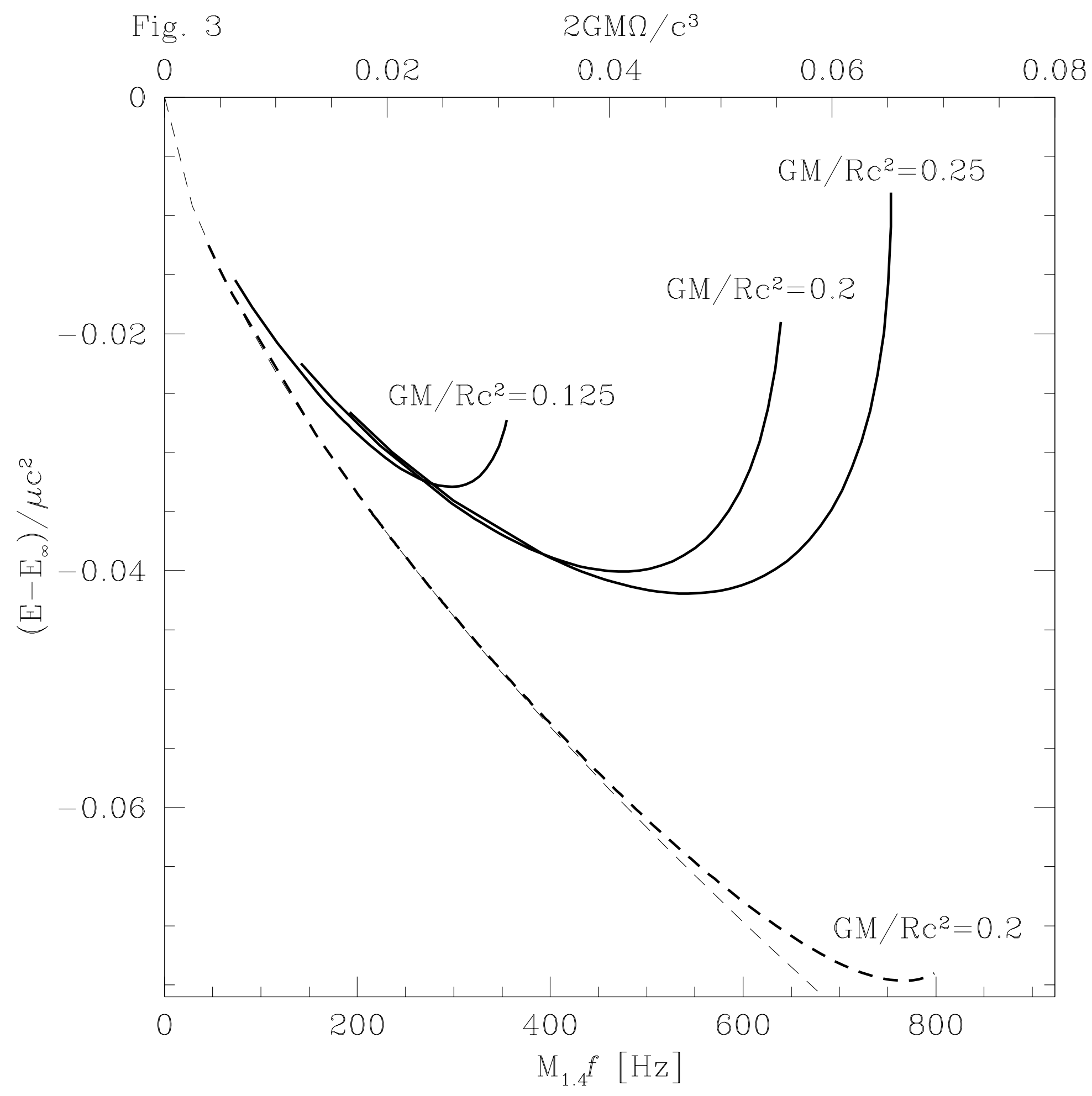


Fig. 4

$2 \mathrm{GM} \Omega_{\mathrm{ISCO}} / \mathrm{C}^{3}$

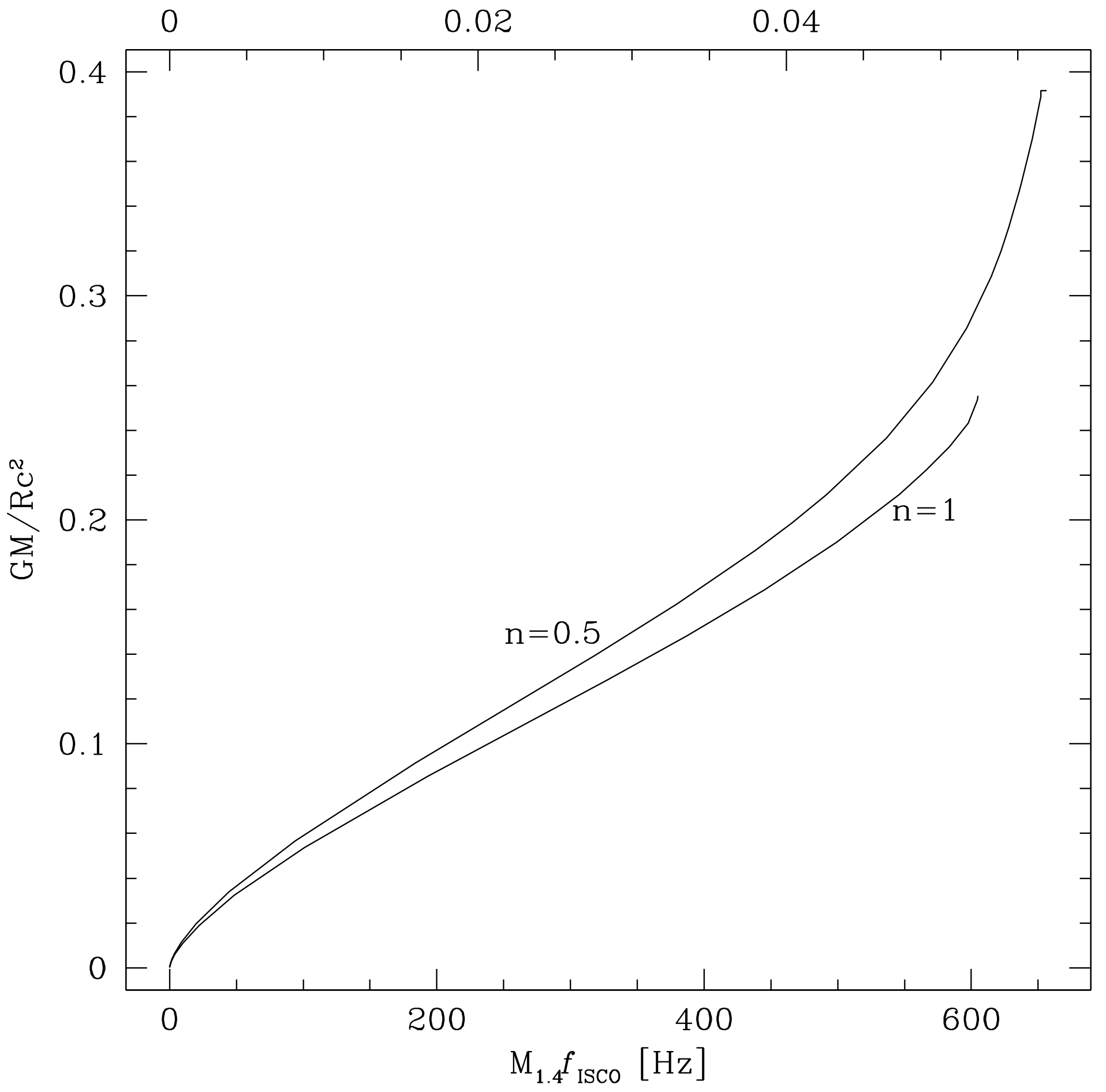




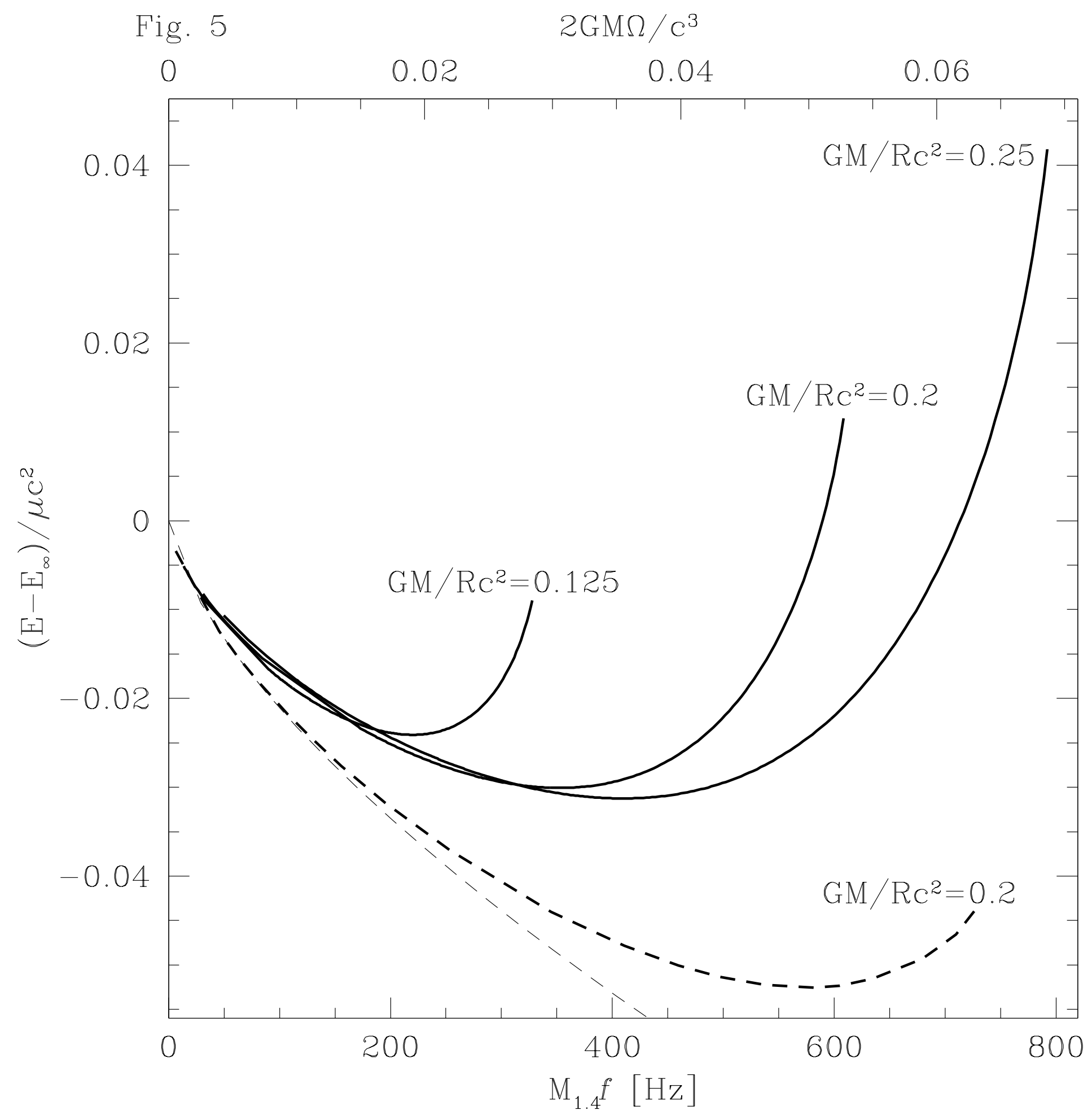




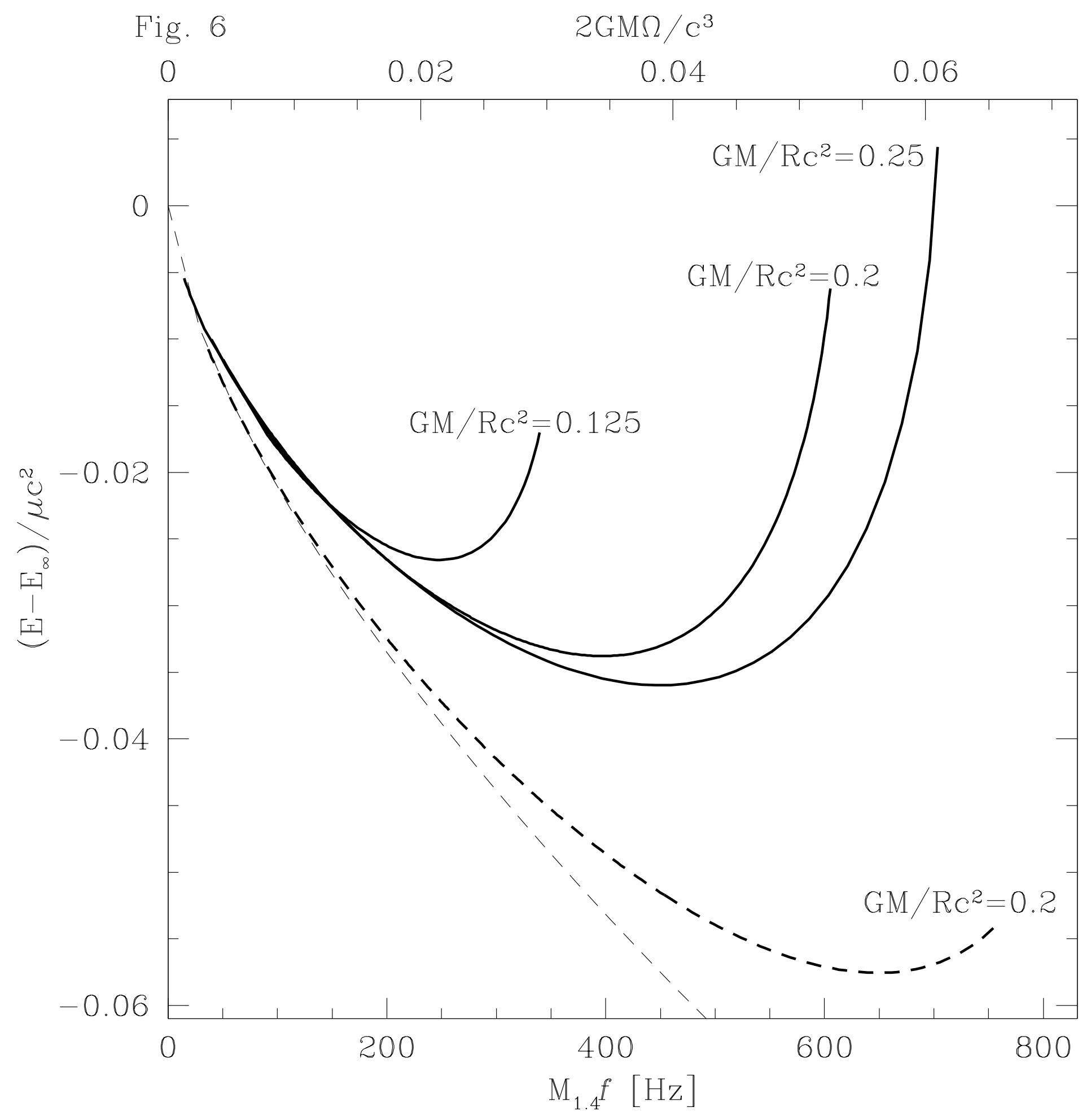


Fig. 7

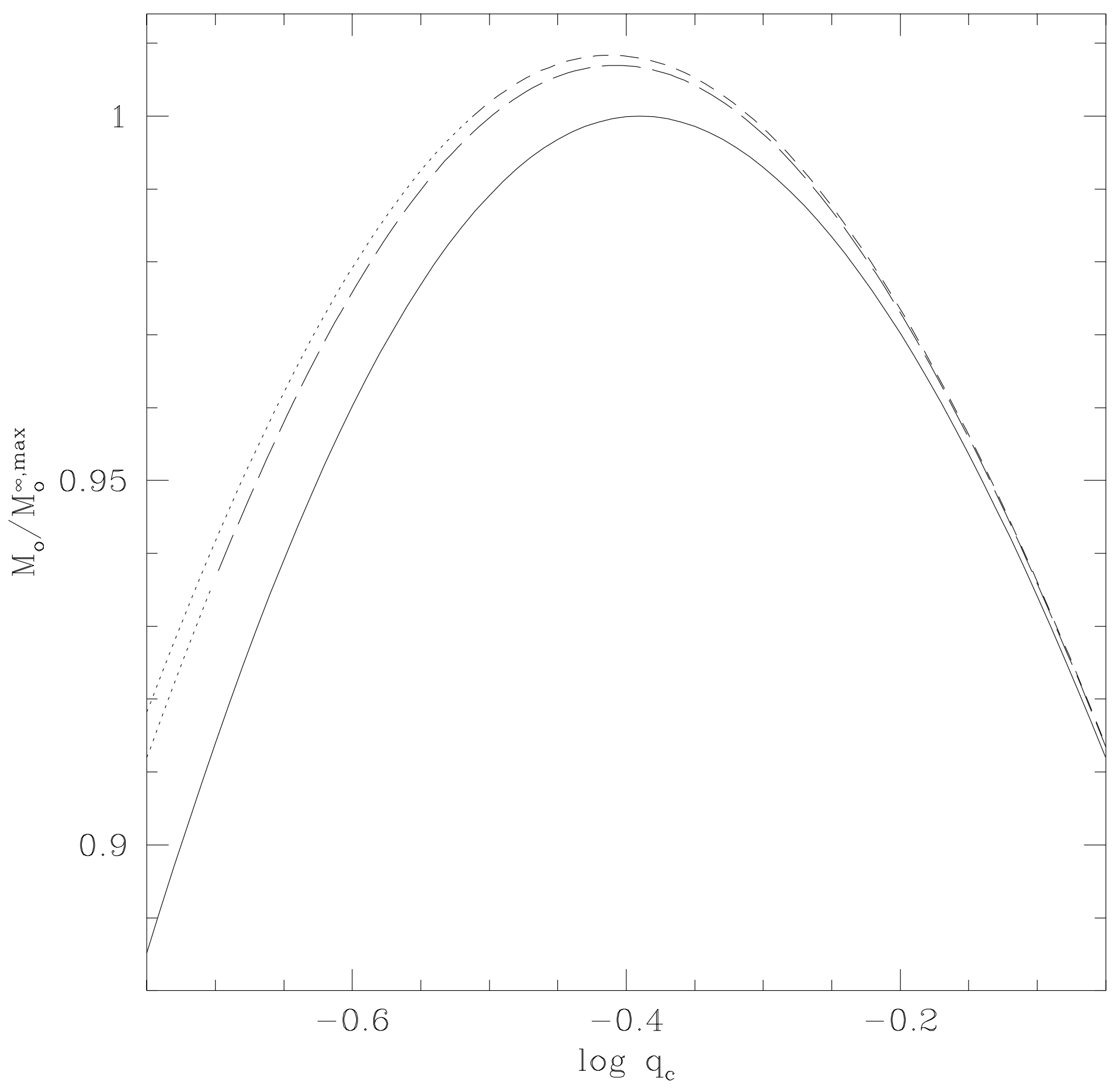


Fig. 8

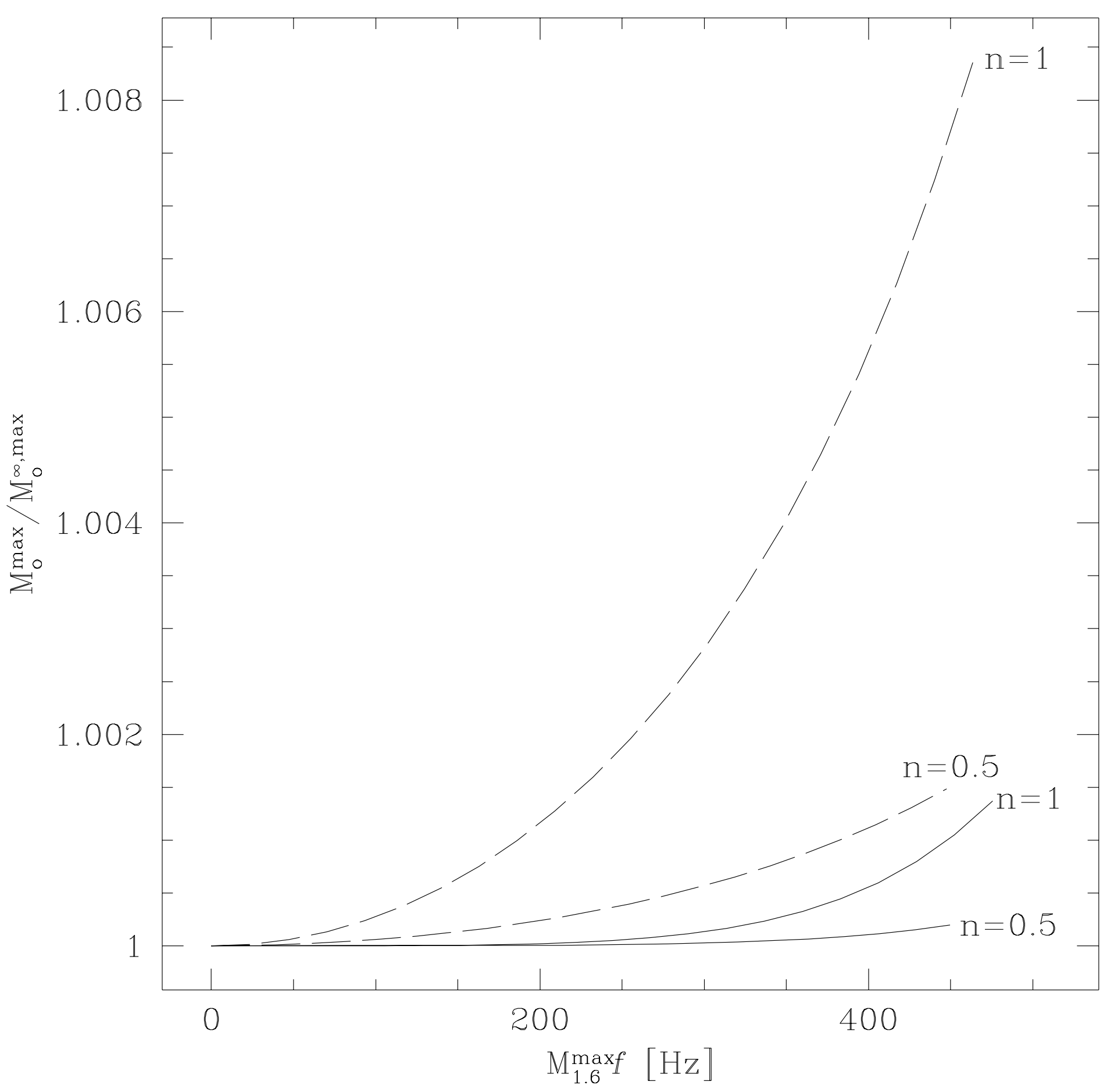


Fig. 9

$2 \mathrm{GM} \Omega / \mathrm{c}^{3}$

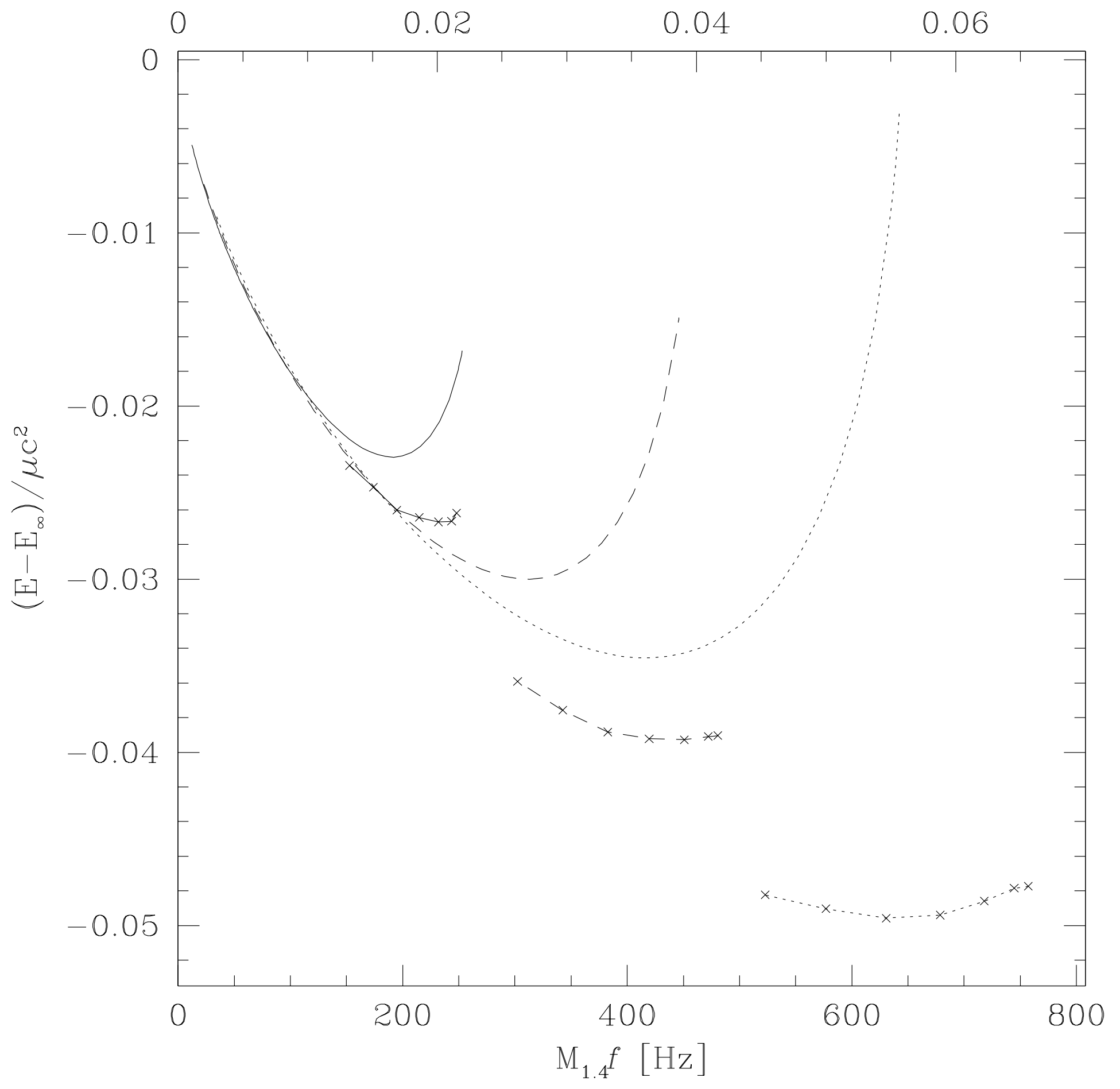

